\title{
HOMOGENIZATION OF THE COMPRESSIBLE NAVIER-STOKES EQUATIONS IN A POROUS MEDIUM
}

\author{
NADER MASMOUdi ${ }^{1}$
}

\begin{abstract}
We study the homogenization of the compressible Navier-Stokes system in a periodic porous medium (of period $\varepsilon$ ) with Dirichlet boundary conditions. At the limit, we recover different systems depending on the scaling we take. In particular, we rigorously derive the so-called "porous medium equation".
\end{abstract}

Mathematics Subject Classification. 76M50.

Received February 5, 2002. Revised March 5, 2002.

\section{INTRODUCTION}

The homogenization of the Stokes and of the incompressible Navier-Stokes equations in a porous medium (open set perforated with tiny holes) has been studied in many works from the formal point of view as well as the rigorous one. We refer the interested reader to $[4,12,20]$ for some formal developments and to $[1,17,21]$ for some rigorous mathematical results. In this paper, we try to extend some of the methods developed in the incompressible case to study the case we start from different compressible models built on the compressible Navier-Stokes system. One of the major difficulties we will encounter here is the passage to the limit in the non linear terms. It is worth noticing that in the incompressible case there are many open problems related to the passage to the limit in the non linear terms due to the presence of boundary layers.

Before stating the system, let us recall the domain we consider. A porous medium is defined as the periodic repetition of an elementary cell of size $\varepsilon$ in a bounded domain $\Omega$ of $\mathbb{R}^{N}$ where $N=2$, or 3 (all the results given below also hold for $N \geq 2$ ). The solid part of the porous medium is also taken of size $\varepsilon$. The domain $\Omega_{\varepsilon}$ is then defined as the intersection of $\Omega$ with the fluid part. We consider a compressible fluid governed by the compressible Navier-Stokes equation. So, we have the following system of equations written in $(0, \infty) \times \Omega_{\varepsilon}$

$$
\left\{\begin{array}{l}
\partial_{t} \rho_{\epsilon}+\operatorname{div}\left(\rho_{\epsilon} u_{\epsilon}\right)=0, \quad \rho_{\epsilon} \geq 0 \\
\partial_{t}\left(\rho_{\epsilon} u_{\epsilon}\right)+\operatorname{div}\left(\rho_{\epsilon} u_{\epsilon} \otimes u_{\epsilon}\right)-\mu \Delta u_{\epsilon}-\xi \nabla \operatorname{div} u_{\epsilon}+\nabla p_{\varepsilon}=\rho_{\varepsilon} f+g
\end{array}\right.
$$

Here, $u_{\varepsilon}$ and $\rho_{\varepsilon}$ are respectively the velocity and the density of the fluid and the pressure $p_{\varepsilon}$ is given by a barotropic law $p_{\varepsilon}=\rho_{\varepsilon}^{\gamma}$. The exterior force is given by $\rho_{\varepsilon} f+g$ and the viscosities $\mu$ and $\xi$ are such that $\mu>0$ and $\mu+\xi>0$. For simplicity, we will assume that $\xi \geq 0$. The system should be complemented with initial and

Keywords and phrases: Compressible Navier-Stokes, homogenization, porous medium equation.

1 Courant Institute, 251 Mercer Street, New York, NY 10012, USA; e-mail: masmoudi@cims.nyu.edu

Partially supported by NSF grant and an Alfred Sloan fellowship. 
boundary conditions

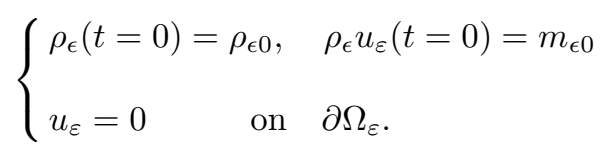

We will study the limit when $\varepsilon$ goes to zero of the above system as well as some related ones with different scalings. The results will be stated and then proved for each model in the next sections.

\subsection{The domain}

Let $\Omega$ be a smooth bounded domain of $\mathbb{R}^{N}$ and define $\left.\mathcal{Y}=\right] 0,1\left[{ }^{N}\right.$ to be the unit open cube of $\mathbb{R}^{N}$. Let $\mathcal{Y}_{s}$ (the solid part) be a closed smooth subset of $\mathcal{Y}$ with a strictly positive measure. The fluid part is then given by $\mathcal{Y}_{f}=\mathcal{Y}-\mathcal{Y}_{s}$ and we define $\theta=\left|\mathcal{Y}_{f}\right|$ the Lebesgue measure of $\mathcal{Y}_{f}$ and we assume that $0<\theta<1$. The constant $\theta$ is called the porosity of the porous medium. Repeating the domain $\mathcal{Y}_{f}$ by $\mathcal{Y}$-periodicity we get the fluid domain $E_{f}$ which can also be defined as

$$
E_{f}=\left\{y \in \mathbb{R}^{N} \mid \exists k \in \mathbb{Z}^{N}, \text { such that } y-k \in \mathcal{Y}_{f}\right\} .
$$

In the same way, we can define $E_{s}=\mathbb{R}^{N}-E_{f}$

$$
E_{s}=\left\{y \in \mathbb{R}^{N} \mid \exists k \in \mathbb{Z}^{N} \text {, such that } y-k \in \mathcal{Y}_{s}\right\} .
$$

It is easy to see that $E_{f}$ is a connected domain, while $E_{s}$ is formed by separate smooth subsets. In the sequel, we denote for all $k \in \mathbb{Z}^{N}, \mathcal{Y}^{k}=\mathcal{Y}+k$ the translate of the cell $\mathcal{Y}$ by the vector $k$, we also denote $\mathcal{Y}_{s}^{k}=\mathcal{Y}_{s}+k$ and $\mathcal{Y}_{f}^{k}=\mathcal{Y}_{f}+k$. Hence, for all $\varepsilon$, we can define the domain $\Omega_{\varepsilon}$ as the intersection of $\Omega$ with the fluid domain rescaled by $\varepsilon$, namely $\Omega_{\varepsilon}=\Omega \cap \varepsilon E_{f}$. However, for some technical reasons and to get a smooth connected domain, we will not remove the solid parts of the cells which intersect the boundary of $\Omega$. We define

$$
\Omega_{\varepsilon}=\Omega-U\left\{\varepsilon \mathcal{Y}_{s}^{k}, \quad \text { where, } k \in \mathbb{Z}^{N}, \varepsilon \mathcal{Y}^{k} \subset \Omega\right\} .
$$

We also denote $K_{\varepsilon}=\left\{k \mid k \in \mathbb{Z}^{N}\right.$ and $\left.\varepsilon \mathcal{Y}^{k} \subset \Omega\right\}$.

Remark 1.1. We can also consider more general domains, specially the more physical case where $E_{s}$ is a connected set of $\mathbb{R}^{N}$ which can be achieved by allowing $\mathcal{Y}_{s}$ to be a closed subset of $\overline{\mathcal{Y}}$ (this is not possible in $N=2$ since we also want that $\Omega_{\varepsilon}$ is connected). We refer the interested reader to the paper of Allaire [1] where the so-called "energy method" of Tartar is extended to the case of a connected $E_{s}$. In the sequel and for the clarity of the presentation, we will only study the case where $E_{s}$ is not connected.

\subsection{Some notations and preliminaries}

In all the paper, we denote the space-time Lebesgue spaces by $L^{r}\left(0, T ; L^{q}(X)\right)$ where $X$ is either $\Omega$ or $\Omega_{\varepsilon}$. Some times we will also denote it by $L_{T}^{r}\left(L^{q}(X)\right), L_{T}^{r}\left(L^{q}\right)$ or $L^{r}\left(L^{q}\right)$ if no ambiguity can occur. If $r=q$, we will also use the notation $L_{T}^{r}(X) . W^{s, p}(X)$ will denote the classical Sobolev space built over $L^{p}$ and $H^{s}(X)=W^{s, 2}(X)$. Besides, we will use the notation $H^{s}(X)^{N}$ (or $H^{s}(X)$ if no ambiguity can occur) for vector valued functions of $N$ components. We will also use Sobolev spaces with negative regularity and we recall that

$$
\|u\|_{W^{-1, p}(X)}=\sup _{v \in W_{0}^{1, p^{\prime}}(X),\|v\|_{W_{0}^{1, p^{\prime}}(X)}}\langle u, v\rangle_{W^{-1, p}, W_{0}^{1, p^{\prime}}}
$$

where $p^{\prime}$ is the conjugate exponent of $p$. 
Due to the presence of the holes $\varepsilon \mathcal{Y}_{s}^{k}$, the domain $\Omega_{\varepsilon}$ depends on $\varepsilon$ and hence to study the convergence of the sequence $\left(u_{\varepsilon}, \rho_{\varepsilon}, p_{\varepsilon}\right)$, we have to extend the functions defined in $\Omega_{\varepsilon}$ to the whole domain $\Omega$. This can be done in two different possible ways.

Definition 1.2. For any function $\phi \in L^{1}\left(\Omega_{\varepsilon}\right)$, we define

$$
\widetilde{\phi}=\left\{\begin{array}{lll}
\phi & \text { in } & \Omega_{\varepsilon} \\
0 & \text { in } & \Omega-\Omega_{\varepsilon}
\end{array}\right.
$$

the extension by 0 of $\phi$ and

$$
\widehat{\phi}=\left\{\begin{array}{lll}
\phi & \text { in } & \Omega_{\varepsilon} \\
\frac{1}{\varepsilon\left|\mathcal{Y}_{f}\right|} \int_{\varepsilon \mathcal{Y}_{f}^{k}} \phi \mathrm{d} y & \text { in } & \varepsilon \mathcal{Y}_{s}^{k}
\end{array} \quad \forall k \in K_{\varepsilon} .\right.
$$

We have the following relation between the weak limits of both types of extensions.

Lemma 1.3. For all sequence $g_{\varepsilon} \in L^{1}\left(\Omega_{\varepsilon}\right)$, the following two assertions are equivalent

1) $\widehat{g}_{\varepsilon} \rightarrow g$ in $L^{1}(\Omega)$;

2) $\widetilde{g}_{\varepsilon} \rightarrow \theta g$ in $L^{1}(\Omega)$.

Proof. For all $\psi \in \mathcal{D}(\Omega)$, we use the fact that $\psi$ is uniformly continuous to deduce that

$$
\omega(\varepsilon)=\sup _{|x-y| \leq \varepsilon}|\psi(x)-\psi(y)| \rightarrow 0 \quad \text { when } \varepsilon \rightarrow 0 .
$$

Hence, we have for $\varepsilon$ small enough

$$
\begin{aligned}
\int_{\Omega} \psi \widetilde{g}_{\varepsilon} & =\sum_{k \in K_{\varepsilon}} \int_{\varepsilon \mathcal{Y}_{f}^{k}} \psi g_{\varepsilon}=\sum_{k \in K_{\varepsilon}} \psi(\varepsilon k) \int_{\varepsilon \mathcal{Y}_{f}^{k}} g_{\varepsilon}+r(\varepsilon) \\
& =\sum_{k \in K_{\varepsilon}} \psi(\varepsilon k) \theta \int_{\varepsilon \mathcal{Y}^{k}} \widehat{g}_{\varepsilon}+r(\varepsilon)=\theta \int_{\Omega} \psi \widehat{g}_{\varepsilon}+r^{\prime}(\varepsilon)
\end{aligned}
$$

where $|r(\varepsilon)|+\left|r^{\prime}(\varepsilon)\right| \leq C \omega(\varepsilon)$. Sending $\varepsilon$ to 0 , we conclude easily.

We will also need the restriction operator constructed by Tartar [21] for the case of a solid part $\mathcal{Y}_{s}$ strictly included in $\mathcal{Y}$ and by Allaire [1] for more general conditions on the solid part.

Lemma 1.4. There exists a linear operator $R_{\varepsilon}$ from $H_{0}^{1}(\Omega)^{N}$ to $H_{0}^{1}\left(\Omega_{\varepsilon}\right)^{N}$ (called restriction operator) such that

(i) $\forall \phi \in H_{0}^{1}\left(\Omega_{\varepsilon}\right)^{N}$, we have $R_{\varepsilon} \widetilde{\phi}=\phi$;

(ii) $\nabla \cdot u=0$ in $\Omega$ implies that $\nabla \cdot R_{\varepsilon} u=0$ in $\Omega_{\varepsilon}$;

(iii) there exists a constant $C$ such that for all $u \in H_{0}^{1}(\Omega)^{N}$, we have

$$
\left\|R_{\varepsilon} u\right\|_{L^{2}\left(\Omega_{\varepsilon}\right)}+\varepsilon\left\|\nabla\left(R_{\varepsilon} u\right)\right\|_{L^{2}\left(\Omega_{\varepsilon}\right)} \leq C\left[\|u\|_{L^{2}(\Omega)}+\varepsilon\|\nabla u\|_{L^{2}(\Omega)}\right] .
$$

The operator $R_{\varepsilon}$ defined above also acts from $W_{0}^{1, r}(\Omega)$ into $W_{0}^{1, r}\left(\Omega_{\varepsilon}\right)$ for all $1<r<\infty$ and we have an estimate similar to (7) where the $L^{2}$ norms are replaced by $L^{r}$ norms.

Due to the presence of the holes in the domain $\Omega_{\varepsilon}$, the Poincaré's inequality reads:

Lemma 1.5. There exists a constant $C$ which depends only on $\mathcal{Y}_{s}$ such that for all $u \in W_{0}^{1, p}\left(\Omega_{\varepsilon}\right)$, we have

$$
\|u\|_{L^{p}\left(\Omega_{\varepsilon}\right)} \leq C \varepsilon\|\nabla u\|_{L^{p}\left(\Omega_{\varepsilon}\right)} .
$$


We refer to [21] for a proof of this lemma. By a simple duality argument we also have the following relation for all $1<p<\infty$

$$
\|u\|_{W^{-1, p}\left(\Omega_{\varepsilon}\right)} \leq C \varepsilon\|u\|_{L^{p}\left(\Omega_{\varepsilon}\right)} .
$$

To get some space-time a priori estimate, we will use the following operator:

Lemma 1.6. For all $\varepsilon>0$, there exists a linear operator $\mathcal{B}=\mathcal{B}_{\varepsilon}$

$$
\mathcal{B}: E_{0}^{p}\left(\Omega_{\varepsilon}\right)=\left\{f \in L^{p}\left(\Omega_{\varepsilon}\right) \mid \int_{\Omega_{\varepsilon}} f=0\right\} \rightarrow W_{0}^{1, p}\left(\Omega_{\varepsilon}\right)
$$

such that $v=\mathcal{B}(f)$ solves the equation

$$
\operatorname{div}(v)=f \quad \text { in } \Omega_{\varepsilon}, \quad v=0 \quad \text { on } \partial \Omega_{\varepsilon}
$$

and the following estimate

$$
\|\mathcal{B}(f)\|_{W_{0}^{1, p}\left(\Omega_{\varepsilon}\right)} \leq \frac{C}{\varepsilon}\|f\|_{L^{p}\left(\Omega_{\varepsilon}\right)}
$$

holds for all $1<p<\infty$. Moreover, if $f \in L^{p}\left(\Omega_{\varepsilon}\right)$ can be written as $f=\operatorname{div}(g)$ where $g \in L^{r}\left(\Omega_{\varepsilon}\right)$ and $g . n=0$ on $\partial \Omega_{\varepsilon}$ for some $r>1$ then

$$
\|\mathcal{B}(f)\|_{L^{r}\left(\Omega_{\varepsilon}\right)} \leq C|| g \|_{L^{r}\left(\Omega_{\varepsilon}\right)}
$$

Sketch of the Proof. The fact that $\mathcal{B}$ maps $L_{0}^{p}\left(\Omega_{\varepsilon}\right)$ into $W_{0}^{1, p}$ is well known (see for instance $\left.[5,10]\right)$. Here we have to explain the presence of the constant $\frac{C}{\varepsilon}$ in the estimate (12). To this end, we will use the construction of Bogovskii. We have to split our domain in small domains and make the construction on each smaller domain. Take an open set $\tilde{\mathcal{Y}}$ such that $\overline{\mathcal{Y}}_{f} \subset \tilde{\mathcal{Y}} \subset E_{f}$ where $\overline{\mathcal{Y}}_{f}=[0,1]^{N}-\mathcal{Y}_{s}$. We define as above, $\tilde{\mathcal{Y}}^{k}=\tilde{\mathcal{Y}}+k$. Then, there exists $\phi \in C_{0}^{\infty}\left(\tilde{\mathcal{Y}} \cup \mathcal{Y}_{s}\right)$ such that

- $\phi=1$ on a neighborhood of $\mathcal{Y}_{s}$;

- $\sum_{k \in \mathbb{Z}^{N}} \phi(x+k)=1 \quad \forall x \in \mathbb{R}^{N}$.

Moreover, for all $k$ and $k^{\prime}$ such that $\left|k-k^{\prime}\right|=1$, we can find a function $\phi_{k, k^{\prime}} \in C_{0}^{\infty}\left(\tilde{\mathcal{Y}}^{k} \cap \tilde{\mathcal{Y}}^{k^{\prime}}\right)$ such that

$$
\int_{\tilde{\mathcal{Y}}^{k} \cap \tilde{\mathcal{Y}}^{k^{\prime}}} \phi_{k, k^{\prime}}=1
$$

Now, for all $f \in L_{0}^{p}\left(\Omega_{\varepsilon}\right)$, we want to construct the solution $v=\mathcal{B}(f)$ by solving an auxiliary problem in each one of the domains $\tilde{\mathcal{Y}}^{k}$. For simplicity, we will assume that $f$ has its support in $U_{k \in K_{\varepsilon}} \mathcal{Y}_{f}^{k}$ to avoid dealing with the part of $f$ close to the boundary of $\Omega$. If we do not make this assumption, we have just to modify the cut-off functions of the cells close to the boundary. Next, we use the partition of the unity to decompose $f$ as $f=\sum_{k \in K_{\varepsilon}} f \phi\left(\frac{x}{\varepsilon}-k\right)$. We note that $f(x) \phi\left(\frac{x}{\varepsilon}-k\right)$ is supported in $\varepsilon \tilde{\mathcal{Y}}^{k}$ but is not necessary of integral equal to 0 . Using the functions $\phi_{k, k^{\prime}}$, we can construct a decomposition $f=\sum_{k \in K_{\varepsilon}} f_{k}$ such that $\operatorname{supp} f_{k} \in \varepsilon \tilde{\mathcal{Y}}^{k}$ and $\int_{\varepsilon \tilde{\mathcal{Y}}^{k}} f_{k}=0$ and $\sum_{k \in K_{\varepsilon}}\left\|f_{k}\right\|_{L^{p}} \leq \frac{C}{\varepsilon}\|f\|_{L^{p}}$. Now, for each $k \in K_{\varepsilon}$, there exists $v_{k} \in W_{0}^{1, p}\left(\varepsilon \tilde{\mathcal{Y}}^{k}\right)$ such that $\operatorname{div} v_{k}=f_{k}$ and $\left.|| v_{k}\right|_{W^{1, p}\left(\varepsilon \tilde{\mathcal{Y}}^{k}\right)} \leq\left.|| v_{k}\right|_{L^{p}\left(\varepsilon \tilde{\mathcal{Y}}^{k}\right)}$. Adding up these estimates, we recover (12). Finally, we point out that in (13) there is no factor $\frac{1}{\varepsilon}$ since we can decompose $g$ as $g=\sum_{k \in K_{\varepsilon}} g_{k}$ where $g_{k}=g \phi\left(\frac{x}{\varepsilon}-k\right)$ and hence we can take $f_{k}=\operatorname{div} g_{k}$. 
For all $\varepsilon>0$, we consider the Stokes problem written in $\Omega_{\varepsilon}$ and define the operator $S=S_{\varepsilon}$ by $S f=p$ where $(u, p)$ solves

$$
\left\{\begin{array}{clll}
-\Delta u+\nabla p=f & \text { in } & \Omega_{\varepsilon} & \\
u=0 & \text { on } & \partial \Omega_{\varepsilon} & \\
\operatorname{div}(u)=0 & \text { in } & \Omega_{\varepsilon} \quad \text { and } \quad \int_{\Omega_{\varepsilon}} p \mathrm{~d} x=0 .
\end{array}\right.
$$

Lemma 1.7. For all $\varepsilon$ and $1<r<\infty$, the operator $S_{\varepsilon}$ is bounded from $L^{r}\left(\Omega_{\varepsilon}\right)$ onto $W^{1, r}\left(\Omega_{\varepsilon}\right)$ and from $W^{-1, r}\left(\Omega_{\varepsilon}\right)$ onto $L^{r}\left(\Omega_{\varepsilon}\right)$ (see for instance [6,10,11,22]). Moreover, there exists a $C$ independent of $\varepsilon$ such that

$$
\begin{gathered}
\|u\|_{H_{0}^{1}\left(\Omega_{\varepsilon}\right)}+\left\|\nabla S_{\varepsilon} f\right\|_{H^{-1}\left(\Omega_{\varepsilon}\right)}+\varepsilon\left\|S_{\varepsilon} f\right\|_{L^{2}\left(\Omega_{\varepsilon}\right)} \leq C\|f\|_{H^{-1}\left(\Omega_{\varepsilon}\right)} \\
\|u\|_{H^{2} \cap H_{0}^{1}\left(\Omega_{\varepsilon}\right)}+\left\|\nabla S_{\varepsilon} f\right\|_{L^{2}\left(\Omega_{\varepsilon}\right)} \leq C\|f\|_{L^{2}\left(\Omega_{\varepsilon}\right)} .
\end{gathered}
$$

Besides, for all $r, 1<r<\infty$ there exists a constant $C$

$$
\begin{gathered}
\|u\|_{W_{0}^{1, r}\left(\Omega_{\varepsilon}\right)}+\left\|\nabla S_{\varepsilon} f\right\|_{W^{-1, r}\left(\Omega_{\varepsilon}\right)}+\varepsilon\left\|S_{\varepsilon} f\right\|_{L^{r}\left(\Omega_{\varepsilon}\right)} \leq \frac{C}{\varepsilon^{\alpha}}\|f\|_{W^{-1, r}\left(\Omega_{\varepsilon}\right)} \\
\|u\|_{W^{2, r} \cap W_{0}^{1, r}\left(\Omega_{\varepsilon}\right)}+\left\|\nabla S_{\varepsilon} f\right\|_{L^{r}\left(\Omega_{\varepsilon}\right)} \leq \frac{C}{\varepsilon^{\alpha}}\|f\|_{L^{r}\left(\Omega_{\varepsilon}\right)}
\end{gathered}
$$

where $\alpha=\left|\frac{N}{2}-\frac{N}{r}\right|$.

Notice here that the factors $\varepsilon^{\alpha}$ associated to $r$ and to the conjugate exponent of $r$ are the same. It is likely that the presence of the factor $1 / \varepsilon^{\alpha}$ is not really necessary in (17) and (18) but we do not need this refinement here.

Sketch of the Proof. Let us start with (15). By the energy estimate, we get

$$
\int_{\Omega_{\varepsilon}}|\nabla u|^{2} \leq C\|f\|_{H^{-1}\left(\Omega_{\varepsilon}\right)}\|u\|_{H_{0}^{1}\left(\Omega_{\varepsilon}\right)} .
$$

From which we deduce that $\|u\|_{H_{0}^{1}\left(\Omega_{\varepsilon}\right)} \leq C\|f\|_{H^{-1}\left(\Omega_{\varepsilon}\right)}$ and that $\|\nabla p\|_{H^{-1}\left(\Omega_{\varepsilon}\right)} \leq C\|f\|_{H^{-1}\left(\Omega_{\varepsilon}\right)}$. Next, using Lemma 1.6, we have

$$
\|p\|_{L^{2}\left(\Omega_{\varepsilon}\right)}^{2}=\langle\nabla p, \mathcal{B}(p)\rangle_{H^{-1}, H_{0}^{1}} \leq \frac{C}{\varepsilon}\|f\|_{H^{-1}\left(\Omega_{\varepsilon}\right)}\|p\|_{L^{2}\left(\Omega_{\varepsilon}\right)} .
$$

Hence (15) is proved. To prove (16), we have to argue as in $[6,10]$ by using interior and boundary regularities and then try to prove that the constants are independent of $\varepsilon$. We start by proving an $H_{0}^{1}$ estimate

$$
\int_{\Omega_{\varepsilon}}|\nabla u|^{2} \leq C\|f\|_{L^{2}\left(\Omega_{\varepsilon}\right)}\|u\|_{L^{2}\left(\Omega_{\varepsilon}\right)} \leq C \varepsilon\|f\|_{L^{2}\left(\Omega_{\varepsilon}\right)}\|u\|_{H_{0}^{1}\left(\Omega_{\varepsilon}\right)} .
$$

From which we deduce that $\|u\|_{H_{0}^{1}\left(\Omega_{\varepsilon}\right)} \leq C \varepsilon\|f\|_{L^{2}\left(\Omega_{\varepsilon}\right)}$ and $\|u\|_{L^{2}\left(\Omega_{\varepsilon}\right)} \leq C \varepsilon^{2}\|f\|_{L^{2}\left(\Omega_{\varepsilon}\right)}$. Now, we will explain the idea behind the uniform bounds, namely the fact that the constant appearing in (16) is independent of $\varepsilon$. Indeed, one can use interior regularity results for each one of the extended cells $\tilde{\mathcal{Y}}^{k}$. We assume that $0 \in \Omega$ and we define $U(x)=u(\varepsilon x), P(x)=p(\varepsilon x), F(x)=f(\varepsilon x)$. Hence $-\Delta U+\varepsilon \nabla P=\varepsilon^{2} F$. Take a cut-off function $\phi \in C_{0}^{\infty}\left(\tilde{\mathcal{Y}} \cup \mathcal{Y}_{s}\right)$ such that $\phi=1$ on a neighborhood of $\mathcal{Y}$, hence

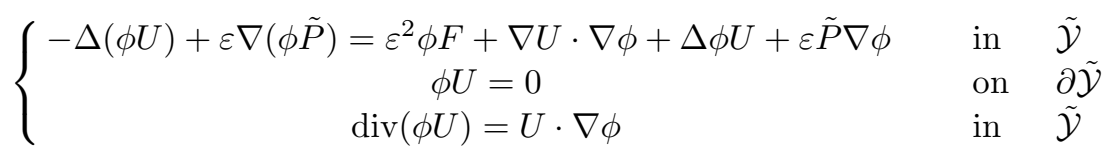


where $\tilde{P}=P-\frac{1}{|\tilde{Y}|} \int_{\tilde{Y}} P$. Using classical regularity results for the Stokes system in a bounded domain, we get that

$$
\begin{aligned}
\|\phi U\|_{H^{2}(\tilde{\mathcal{Y}})}+\|\varepsilon \nabla(\phi \tilde{P})\|_{L^{2}(\tilde{\mathcal{Y}}) \leq} & C\|U \cdot \nabla \phi\|_{L^{2}(\tilde{\mathcal{Y}})}+C\left\|\varepsilon^{2} \phi F\right\|_{L^{2}(\tilde{\mathcal{Y}})} \\
& +C\|\nabla U \cdot \nabla \phi+\Delta \phi U+\varepsilon \tilde{P} \nabla \phi\|_{L^{2}(\tilde{\mathcal{Y}})} \\
\leq & C\left[\left\|\varepsilon^{2} F\right\|_{L^{2}(\tilde{\mathcal{Y}})}+\|\nabla U\|_{L^{2}(\tilde{\mathcal{Y}})}+\|U\|_{L^{2}(\tilde{\mathcal{Y}})}+\varepsilon\|\tilde{P}\|_{L^{2}(\tilde{\mathcal{Y}})}\right] .
\end{aligned}
$$

Besides, we have

$$
\|\varepsilon \tilde{P}\|_{L^{2}(\tilde{\mathcal{Y}})} \leq C\|\varepsilon \nabla \tilde{P}\|_{H^{-1}(\tilde{\mathcal{Y}})} \leq C\left\|\varepsilon^{2} F\right\|_{H^{-1}(\tilde{\mathcal{Y}})}+C\|U\|_{H^{1}(\tilde{\mathcal{Y}})}
$$

Hence, the last term in (20) can be estimated by the other terms appearing in the right hand side. Rewriting (20) in the original coordinate system, we get

$$
\|u\|_{H^{2}\left(\varepsilon \mathcal{Y}_{f}\right)}+\|\nabla p\|_{L^{2}\left(\varepsilon \mathcal{Y}_{f}\right)} \leq C\|f\|_{L^{2}(\varepsilon \tilde{\mathcal{Y}})}+\frac{C}{\varepsilon}\|\nabla u\|_{L^{2}(\varepsilon \tilde{\mathcal{Y}})}+\frac{C}{\varepsilon^{2}}\|u\|_{L^{2}(\varepsilon \tilde{\mathcal{Y}})} .
$$

This estimate also hold for any cell $\varepsilon \mathcal{Y}_{f}$ such that $\varepsilon \tilde{\mathcal{Y}} \subset \Omega_{\varepsilon}$. Near the boundary the above argument should be slightly changed. Adding up the above estimates, we infer

$$
\|u\|_{H^{2}\left(\Omega_{\varepsilon}\right)}+\|\nabla p\|_{L^{2}\left(\Omega_{\varepsilon}\right)} \leq C\|f\|_{L^{2}\left(\Omega_{\varepsilon}\right)}+\frac{C}{\varepsilon}\|\nabla u\|_{L^{2}\left(\Omega_{\varepsilon}\right)}+\frac{C}{\varepsilon^{2}}\|u\|_{L^{2}\left(\Omega_{\varepsilon}\right)} .
$$

From which we deduce (16).

To prove (17), we restrict ourselves to the case $r>2$ since the case $r<2$ can be deduce from the case $r>2$ by duality. Using that $W^{-1, r} \subset H^{-1}$ since $r>2$, we deduce that $\|u\|_{H_{0}^{1}\left(\Omega_{\varepsilon}\right)} \leq C\|f\|_{W^{-1, r}\left(\Omega_{\varepsilon}\right)}$ and that $\|\nabla p\|_{H^{-1}\left(\Omega_{\varepsilon}\right)} \leq C\|f\|_{W^{-1, r}\left(\Omega_{\varepsilon}\right)}$. Moreover, arguing as above, we have

$$
\begin{aligned}
\|\phi U\|_{W^{1, r}(\tilde{\mathcal{Y}})}+\|\varepsilon \nabla(\phi \tilde{P})\|_{W^{-1, r}(\tilde{\mathcal{Y}}) \leq} & C\|U \cdot \nabla \phi\|_{W^{-1, r}(\tilde{\mathcal{Y}})} C\left\|\varepsilon^{2} \phi F\right\|_{W^{-1, r}(\tilde{\mathcal{Y}})} \\
& +C\|\nabla U \cdot \nabla \phi+\Delta \phi U+\varepsilon \tilde{P} \nabla \phi\|_{W^{-1, r}(\tilde{\mathcal{Y}})} \\
\leq & C\left[\left\|\varepsilon^{2} F\right\|_{W^{-1, r}}+\|\nabla U\|_{W^{-1, r}}+\|U\|_{W^{-1, r}}+\varepsilon\|\tilde{P}\|_{W^{-1, r}}\right] .
\end{aligned}
$$

Besides, we have

$$
\|\varepsilon \tilde{P}\|_{W^{-1, r}(\tilde{\mathcal{Y}})} \leq C\|\varepsilon \nabla \tilde{P}\|_{W^{-2, r}(\tilde{\mathcal{Y}})} \leq C\left\|\varepsilon^{2} F\right\|_{W^{-2, r}(\tilde{\mathcal{Y}})}+C\|U\|_{L^{r}(\tilde{\mathcal{Y}})}
$$

and $\|\nabla U\|_{W^{-1, r}}+\|U\|_{W^{-1, r}} \leq C|| U \|_{L^{r}(\tilde{\mathcal{Y}})}$. Adding up all the estimates over the different cells and going back to the initial coordinate system, we get

$$
\|u\|_{W_{0}^{1, r}\left(\Omega_{\varepsilon}\right)}+\|\nabla p\|_{W^{-1, r}\left(\Omega_{\varepsilon}\right)} \leq C\|f\|_{W^{-1, r}}+\frac{C}{\varepsilon}\|u\|_{L^{r}\left(\Omega_{\varepsilon}\right)} .
$$

Next, we use that

$$
\|u\|_{L^{r}\left(\Omega_{\varepsilon}\right)} \leq C\|u\|_{L^{2}\left(\Omega_{\varepsilon}\right)}^{\kappa} \mid\|\nabla u\|_{L^{r}\left(\Omega_{\varepsilon}\right)}^{1-\kappa}
$$

where $\frac{1}{r}=\frac{\kappa}{2}+(1-\kappa)\left(\frac{1}{r}-\frac{1}{N}\right)$. Hence, we get that

$$
\|u\|_{W_{0}^{1, r}\left(\Omega_{\varepsilon}\right)} \leq C\|f\|_{W^{-1, r}}+\frac{C}{\varepsilon}\|u\|_{L^{2}\left(\Omega_{\varepsilon}\right)}^{\kappa}\|\nabla u\|_{L^{r}\left(\Omega_{\varepsilon}\right)}^{1-\kappa}
$$


which can be rewritten as

$$
\|u\|_{W_{0}^{1, r}\left(\Omega_{\varepsilon}\right)} \leq C\|f\|_{W^{-1, r}}+\frac{C}{\varepsilon^{\frac{1}{\kappa}}}\|u\|_{L^{2}\left(\Omega_{\varepsilon}\right)} \leq \frac{C}{\varepsilon^{\frac{1}{\kappa}-1}}\|f\|_{W^{-1, r}} .
$$

And (17) is proved, since $\frac{1}{\kappa}-1=\alpha$.

Finally, we define the permeability matrix $\bar{A}$. For all $i, 1 \leq i \leq N$, let $\left(v_{i}, q_{i}\right) \in H^{1}\left(\mathcal{Y}_{f}\right)^{N} \times L^{2}\left(\mathcal{Y}_{f}\right) / \mathbb{R}$ be the unique solution of the following system

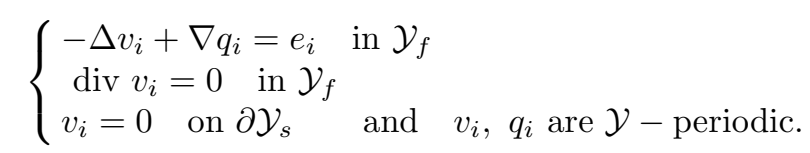

Using regularity results of the Stokes problem, we infer that $v_{i}$ and $q_{i}$ are smooth. We extend $v_{i}$ to the whole domain $\mathcal{Y}$ by setting $v_{i}(y)=0$ if $y \in \mathcal{Y}_{s}$. Then, for all $y \in \mathcal{Y}_{f}, A(y)$ is taken to be the matrix composed of the column vectors $v_{i}(y)$ and $\bar{A}=\int_{\mathcal{Y}_{f}} A(y) \mathrm{d} y$. It is easy to see that $\bar{A}$ is a symmetric positive definite matrix. Indeed, multiplying the first equation in $\left(S_{i}\right)$ by $v_{j}$ and the first equation in $\left(S_{j}\right)$ by $v_{i}$, we get that $\int_{\mathcal{Y}_{f}} \nabla v_{i} \cdot \nabla v_{j}=\int_{\mathcal{Y}_{f}} v_{j i}=\bar{A}_{j i}$ and $\int_{\mathcal{Y}_{f}} \nabla v_{j} \cdot \nabla v_{i}=\int_{\mathcal{Y}_{f}} v_{i j}=\bar{A}_{i j}$ where we wrote $v_{i}(y)=\sum_{j=1}^{N} v_{j i}(y) e_{j}$. Then to prove that $\bar{A}$ is positive definite, we just notice that for all vector $X=\sum_{j=1}^{N} x_{i} e_{i}$, we have $\sum_{i j} x_{i} \bar{A}_{i j} x_{j}=$ $\left\|\nabla \sum_{j=1}^{N} x_{j} v_{j}\right\|_{L^{2}\left(\mathcal{Y}_{f}\right)}^{2}$ and that $\left\{v_{i}, 1 \leq i \leq N\right\}$ is an independent family.

In the next three sections we will study three different types of models. For each model, we will start by a presentation then state the result and finally give the proof of the main result. In Section 2, we study a semi-stationary model and derive in particular the so-called "porous medium" equation. In Section 3, we start from the full compressible system but with a scaling which gives formally the same limit system as in Section 2 . Finally, in Section 4, we deal with an equation describing the acoustics in a porous medium.

\section{A SEMI-STATIONARY MODEL}

\subsection{The model}

We start with the following semi-stationary model

$$
\left\{\begin{array}{l}
\varepsilon^{2} \partial_{t} \rho_{\epsilon}+\operatorname{div}\left(\rho_{\epsilon} u_{\epsilon}\right)=0, \\
-\mu \Delta u_{\epsilon}-\xi \nabla \operatorname{div} u_{\epsilon}+\nabla \rho_{\epsilon}^{\gamma}=\rho_{\varepsilon} f+g
\end{array}\right.
$$

complemented with the boundary condition $u_{\varepsilon}=0$ on $\partial \Omega_{\varepsilon}$ and the initial condition $\rho_{\varepsilon}(t=0)=\rho_{\varepsilon 0}$. The force term is such that $f \in L^{\infty}\left((0, T) \times \Omega_{\varepsilon}\right)$ and $g \in L^{2}\left((0, T) \times \Omega_{\varepsilon}\right)$. We also assume that $\gamma \geq 1$ and that $\|f\|_{L^{\infty}}$ is small enough if $\gamma=1$.

\subsection{Statement of the result}

We assume that the initial data is such that $\rho_{\varepsilon 0} \in L^{1} \cap L^{\gamma}\left(\Omega_{\varepsilon}\right)$ if $\gamma>1$, that $\int_{\Omega_{\varepsilon}} \rho_{\varepsilon 0}\left|\log \rho_{\varepsilon 0}\right|<C$ if $\gamma=1$ and that $\widehat{\rho}_{\varepsilon 0}$ converges weakly to $\rho_{0}$ in $L^{\gamma}(\Omega)$.

We consider a sequence of weak solutions $\left(\rho_{\varepsilon}, u_{\varepsilon}\right)$ of the semi-stationary model (27) such that for all $T>0$, $\rho_{\varepsilon} \in C\left([0, T) ; L^{1}\left(\Omega_{\varepsilon}\right)\right) \cap L^{\infty}\left(0, T ; L^{\gamma}\left(\Omega_{\varepsilon}\right)\right) \cap L^{2 \gamma}\left((0, T) \times \Omega_{\varepsilon}\right)$ and $\rho_{\varepsilon}\left|\log \rho_{\varepsilon}\right| \in L^{\infty}\left(0, T ; L^{1}\left(\Omega_{\varepsilon}\right)\right)$ if $\gamma=1$. Moreover, $u_{\varepsilon}$ is such that $\frac{u_{\varepsilon}}{\varepsilon} \in L^{2}\left(0, T ; H_{0}^{1}\left(\Omega_{\varepsilon}\right)\right)$ and $\frac{u_{\varepsilon}}{\varepsilon^{2}} \in L^{2}\left((0, T) \times \Omega_{\varepsilon}\right)$. Finally, we also require that $\widehat{p}_{\varepsilon}$ is bounded in $L_{T}^{2}\left(H^{1}(\Omega)\right)+\varepsilon L_{T}^{2}\left(L^{2}(\Omega)\right)$. We assume that the bounds given above are uniform in $\varepsilon$. We point out that the 
fact that we can consider a sequence of solutions satisfying the above uniform estimates is a consequence of the a priori bounds which will be recalled in Section 2.3 .

Before studying the limit of the sequence $\left(u_{\varepsilon}, \rho_{\varepsilon}, p_{\varepsilon}\right)$, we have to prolong it to $\Omega$. Let $\widetilde{u}_{\varepsilon}, \widetilde{\rho}_{\varepsilon}$ and $\widehat{p}_{\varepsilon}$ be the extensions of $u_{\varepsilon}, \rho_{\varepsilon}$ and $p_{\varepsilon}$ to the whole domain $\Omega$ defined as in Section 1.2.

Theorem 2.1. Under the above assumptions,

$$
\begin{aligned}
& \widetilde{\rho}_{\varepsilon} \rightarrow \theta \rho \quad \text { weakly in } L_{T}^{r}\left(L^{\gamma}(\Omega)\right) \cap L^{2 \gamma}((0, T) \times \Omega), \\
& \widehat{\rho}_{\varepsilon} \rightarrow \quad \rho \quad \text { strongly in } L_{T}^{r}\left(L^{\gamma}(\Omega)\right) \cap L^{\gamma+1}((0, T) \times \Omega), \\
& \frac{\widetilde{u}_{\varepsilon}}{\varepsilon^{2}} \rightarrow u \quad \text { weakly in } L_{T}^{2}\left(L^{2}(\Omega)\right)
\end{aligned}
$$

for all $r<\infty$ where $\rho \in L^{2 \gamma}((0, T) \times \Omega), \rho^{\gamma} \in L_{T}^{2}\left(H^{1}(\Omega)\right)$ and $\rho$ is the solution of the following system

$$
\left\{\begin{array}{l}
\theta \partial_{t} \rho+\frac{1}{\mu} \operatorname{div} \cdot\left[\rho \bar{A}\left(\rho f+g-\nabla \rho^{\gamma}\right)\right]=0 \\
\rho \bar{A}\left(\rho f+g-\nabla \rho^{\gamma}\right) \cdot n=0 \quad \text { on } \quad \partial \Omega \\
\rho(t=0)=\rho_{0}
\end{array}\right.
$$

and $u$ is given by

$$
u=\bar{A}\left(\rho f+g-\nabla \rho^{\gamma}\right) \quad \text { on } \quad\{\rho>0\}
$$

We point out here that even though each one of the terms $f, g$ and $\nabla \rho^{\gamma}$ does not have necessary a trace on the boundary $\partial \Omega$, the combination of them appearing in (28) has a sense. A formal derivation of the system (28) can be found in [8]. The relation (29) giving $u$ as a function of the pressure is a Darcy law [7].

Remark 2.2. if $\bar{A}=\alpha I$ (which is the case if for instance $\mathcal{Y}_{s}$ is a ball) and $f=g=0$ then we get the following system

$$
\left\{\begin{array}{l}
\partial_{t} \rho-\beta \Delta \rho^{\gamma+1}=0 \\
\frac{\partial \rho^{\gamma+1}}{\partial n}=0 \text { on } \quad \partial \Omega \\
\rho(t=0)=\rho_{0}
\end{array}\right.
$$

where $\beta=\frac{\alpha \gamma}{\theta \mu(\gamma+1)}$. This system is the so-called "porous medium" equation.

\subsection{A priori estimates}

Before proving Theorem 2.1, let us recall how we can get the existence of weak solutions for (27) satisfying the requirement of the last subsection. We will only explain how we can get uniform estimates in $\varepsilon$ and refer to [14] (p. 226) for the approximation part. First, integrating the first equation of (27) over the whole domain $\Omega_{\varepsilon}$, we deduce the conservation of mass, namely $\int_{\Omega_{\varepsilon}} \rho_{\varepsilon}=\int_{\Omega_{\varepsilon}} \rho_{\varepsilon 0}$. The fact that we can make this integration over the whole domain $\Omega_{\varepsilon}$ rigorously comes from the $L^{2}$ bounds we have for $\rho_{\varepsilon}$ and $\nabla u_{\varepsilon}$. Then, multiplying the second 
equation of $(27)$ by $u_{\varepsilon}$ and using the first one, we get (at least formly and in the case $\gamma>1$ ) the following equality for all $t>0$

$$
\varepsilon^{2} \int_{\Omega_{\varepsilon}} \frac{\rho_{\varepsilon}^{\gamma}(t)}{\gamma-1}+\int_{0}^{t} \int_{\Omega_{\varepsilon}} \mu\left|\nabla u_{\varepsilon}\right|^{2}+\xi\left(\operatorname{div} u_{\varepsilon}\right)^{2}=\varepsilon^{2} \int_{\Omega_{\varepsilon}} \frac{\rho_{\varepsilon 0}^{\gamma}}{\gamma-1}+\int_{0}^{t} \int_{\Omega_{\varepsilon}}\left(\rho_{\varepsilon} f+g\right) \cdot u_{\varepsilon}
$$

while if $\gamma=1$, we get

$$
\varepsilon^{2} \int_{\Omega_{\varepsilon}} \rho_{\varepsilon} \log \rho_{\varepsilon}(t)+\int_{0}^{t} \int_{\Omega_{\varepsilon}} \mu\left|\nabla u_{\varepsilon}\right|^{2}+\xi\left(\operatorname{div} u_{\varepsilon}\right)^{2}=\varepsilon^{2} \int_{\Omega_{\varepsilon}} \rho_{\varepsilon 0} \log \rho_{\varepsilon 0}+\int_{0}^{t} \int_{\Omega_{\varepsilon}}\left(\rho_{\varepsilon} f+g\right) \cdot u_{\varepsilon} .
$$

We start with the case $\gamma \geq 2$, then we can estimate the right hand side

$$
\begin{aligned}
\int_{\Omega_{\varepsilon}}\left|\left(\rho_{\varepsilon} f+g\right) \cdot u_{\varepsilon}\right| & \leq \varepsilon^{2} \frac{C}{\mu}\left(\|f\|_{L^{\infty}}^{2}\left\|\rho_{\varepsilon}\right\|_{L^{2}\left(\Omega_{\varepsilon}\right)}^{2}+\|g\|_{L^{2}\left(\Omega_{\varepsilon}\right)}^{2}\right)+\frac{\mu}{2 C \varepsilon^{2}}\left\|u_{\varepsilon}\right\|_{L^{2}\left(\Omega_{\varepsilon}\right)}^{2} \\
& \leq \varepsilon^{2} \frac{C}{\mu}\left(\|f\|_{L^{\infty}}^{2}\left\|\rho_{\varepsilon}\right\|_{L^{2}\left(\Omega_{\varepsilon}\right)}^{2}+\|g\|_{L^{2}\left(\Omega_{\varepsilon}\right)}^{2}\right)+\frac{\mu}{2}\left\|\nabla u_{\varepsilon}\right\|_{L^{2}\left(\Omega_{\varepsilon}\right)}^{2}
\end{aligned}
$$

where $C$ is the constant appearing in (8). Hence, we deduce that for all $T$

$$
\varepsilon^{2} \int_{\Omega_{\varepsilon}} \frac{\rho_{\varepsilon}^{\gamma}(T)}{\gamma-1}+\int_{0}^{T} \int_{\Omega_{\varepsilon}} \frac{\mu}{2}|\nabla u|^{2} \leq \varepsilon^{2} \int_{\Omega_{\varepsilon}} \frac{\rho_{\varepsilon}^{\gamma}}{\gamma-1}+\frac{C \varepsilon^{2}}{\mu}\left(\|f\|_{L^{\infty}}^{2}\left\|\rho_{\varepsilon}\right\|_{L^{2}\left((0, T) \times \Omega_{\varepsilon}\right)}^{2}+\|g\|_{L^{2}\left((0, T) \times \Omega_{\varepsilon}\right)}^{2}\right) .
$$

Then using Gronwall lemma and the fact that $\left\|\rho_{\varepsilon}\right\|_{L^{2}\left(\Omega_{\varepsilon}\right)}^{2} \leq C\left(1+\left\|\rho_{\varepsilon}\right\|_{L^{\gamma}\left(\Omega_{\varepsilon}\right)}^{\gamma}\right)$, we deduce that for all $T$ there exists a constant $C_{T}$ such that

$$
\sup _{0 \leq t \leq T} \int_{\Omega_{\varepsilon}} \rho_{\varepsilon}^{\gamma}(t)+\int_{0}^{T} \int_{\Omega_{\varepsilon}}\left|\nabla \frac{u_{\varepsilon}}{\varepsilon}\right|^{2} \leq C_{T}
$$

Then, from the second equation of (27), we want to deduce a uniform bound for $p_{\varepsilon}=\rho_{\varepsilon}^{\gamma}$ in $L^{2}\left((0, T) \times \Omega_{\varepsilon}\right)$. This can be done using Lemma 1.6, however to get some compactness in space, we will use another method based on the extension of $\nabla p_{\varepsilon}$ to the whole domain $\Omega$. This is done using an extension operator which is the dual of the restriction operator $R_{\varepsilon}$ defined in Lemma 1.4. Let $F_{\varepsilon} \in L_{T}^{2}\left(H^{-1}(\Omega)\right)$ be defined by

$$
\int_{0}^{T}\left\langle F_{\varepsilon}, v\right\rangle_{H^{-1}, H_{0}^{1}(\Omega)}=\int_{0}^{T}\left\langle\nabla p_{\varepsilon}, R_{\varepsilon}(v)\right\rangle_{H^{-1}, H_{0}^{1}\left(\Omega_{\varepsilon}\right)} \quad \forall v \in L_{T}^{2}\left(H_{0}^{1}(\Omega)\right) .
$$

Using that

$$
\begin{aligned}
\int_{0}^{T}\left\langle\nabla p_{\varepsilon}, R_{\varepsilon}(v)\right\rangle_{H^{-1}, H_{0}^{1}\left(\Omega_{\varepsilon}\right)} & =\int_{0}^{T}\left\langle\mu \Delta u_{\varepsilon}+\xi \nabla \operatorname{div} u_{\varepsilon}+\rho_{\varepsilon} f+g, R_{\varepsilon} v\right\rangle_{H^{-1}, H_{0}^{1}\left(\Omega_{\varepsilon}\right)} \\
& =\int_{0}^{T} \int_{\Omega_{\varepsilon}}-\mu \nabla u_{\varepsilon} \cdot \nabla R_{\varepsilon}(v)-\xi \operatorname{div} u_{\varepsilon} \operatorname{div} v+\left(\rho_{\varepsilon} f+g\right) R_{\varepsilon} v
\end{aligned}
$$

we obtain

$$
\begin{aligned}
\left|\int_{0}^{T}\left\langle F_{\varepsilon}, v\right\rangle_{H^{-1}, H_{0}^{1}(\Omega)}\right| \leq & C\left[\frac{1}{\varepsilon}\left\|\nabla u_{\varepsilon}\right\|_{L_{T}^{2}\left(\Omega_{\varepsilon}\right)}+\|f\|_{L^{\infty}}\|\rho\|_{L_{T}^{2}\left(\Omega_{\varepsilon}\right)}+\|g\|_{L_{T}^{2}\left(\Omega_{\varepsilon}\right)}\right] \\
\times & {\left[\|v\|_{L_{T}^{2}(\Omega)}+\varepsilon\|\nabla v\|_{L_{T}^{2}(\Omega)}\right] . }
\end{aligned}
$$


Hence, we deduce that $F_{\varepsilon}$ is bounded in $L_{T}^{2}\left(L^{2}(\Omega)\right)+\varepsilon L_{T}^{2}\left(H^{-1}(\Omega)\right.$ ). Moreover property (ii) of Lemma 1.4 implies that there exists a $P_{\varepsilon} \in L_{T}^{2}\left(L^{2}(\Omega)\right)$ such that $F_{\varepsilon}=\nabla P_{\varepsilon}$ and from the bound on $F_{\varepsilon}$, we get that $P_{\varepsilon}$ is bounded in $L_{T}^{2}\left(H^{1}(\Omega)\right)+\varepsilon L_{T}^{2}\left(L^{2}(\Omega)\right)$. A result of Lipton and Avellaneda [15] (see also Allaire [1]) shows that up to a constant, we have $P_{\varepsilon}=\widehat{p}_{\varepsilon}$.

Now, let us concentrate on the case $1 \leq \gamma<2$. If $f=0$ then we can argue exactly as above. if $f \neq 0$, then we have to combine (34) with the estimate based on the space-time integrability of the pressure $\widehat{p}_{\varepsilon}$. Arguing as above, we deduce from (37) that

$$
\left\|\widehat{p}_{\varepsilon}\right\|_{L_{T}^{2}\left(H^{1}(\Omega)\right)+\varepsilon L_{T}^{2}\left(L^{2}(\Omega)\right)} \leq C\left[\frac{1}{\varepsilon}\left\|\nabla u_{\varepsilon}\right\|_{L_{T}^{2}\left(\Omega_{\varepsilon}\right)}+\|f\|_{L^{\infty}}\|\rho\|_{L_{T}^{2}\left(\Omega_{\varepsilon}\right)}+\|g\|_{L_{T}^{2}\left(\Omega_{\varepsilon}\right)}\right] .
$$

Combining (38) with (34), we infer that

$$
\left\|\widehat{p}_{\varepsilon}\right\|_{L_{T}^{2}\left(L^{2}(\Omega)\right)} \leq C\left[1+\|f\|_{L^{\infty}}\|\rho\|_{L_{T}^{2}\left(\Omega_{\varepsilon}\right)}+\|g\|_{L^{2}\left(\Omega_{\varepsilon}\right)}\right]
$$

which can be rewritten

$$
\left\|\widehat{p}_{\varepsilon}\right\|_{L_{T}^{2}\left(L^{2}(\Omega)\right)} \leq C_{T}+C|| \widehat{p}_{\varepsilon} \|_{L_{T}^{2}\left(L^{2}(\Omega)\right)}^{\frac{2}{\gamma+1}} .
$$

Hence, we deduce a bound for $\rho^{2 \gamma}$ if $\gamma>1$. In the case $\gamma=1$, we use the smallness condition on $f$ in the $L^{\infty}$ norm to make the constant $C$ appearing in (40) smaller than 1 and then deduce a bound for $\widehat{\rho}_{\varepsilon}$ in $L_{T}^{2}\left(L^{2}(\Omega)\right)$.

In all cases, namely $\gamma \geq 1$, we deduce that for all $T$, there exists a constant $C_{T}$ such that

$$
\sup _{0 \leq t \leq T} \int_{\Omega_{\varepsilon}} \rho_{\varepsilon}^{\gamma}(t)+\int_{0}^{T} \int_{\Omega_{\varepsilon}} \rho_{\varepsilon}^{2 \gamma}+\frac{1}{\varepsilon^{2}} \int_{0}^{T} \int_{\Omega_{\varepsilon}}\left|\nabla u_{\varepsilon}\right|^{2} \leq C_{T}
$$

as well as the fact that $\widehat{p}_{\varepsilon}$ is bounded in $L_{T}^{2}\left(H^{1}(\Omega)\right)+\varepsilon L_{T}^{2}\left(L^{2}(\Omega)\right)$.

\subsection{Convergence proof}

Using that $\widetilde{\rho}_{\varepsilon}$ and $\widehat{\rho}_{\varepsilon}$ are bounded in $L^{\infty}\left(0, T ; L^{\gamma}(\Omega)\right) \cap L^{2 \gamma}((0, T) \times \Omega)$, we can extract subsequences (still denoted $\widetilde{\rho}_{\varepsilon}$ and $\left.\widehat{\rho}_{\varepsilon}\right)$ such that $\widehat{\rho}_{\varepsilon}$ converges weakly to some $\rho$ where $\rho \in L^{\infty}\left(0, T ; L^{\gamma}(\Omega)\right) \cap L^{2 \gamma}((0, T) \times \Omega)$ and $\widetilde{\rho}_{\varepsilon}$ converges weakly to $\theta \rho$. Besides, using that $\left\|\frac{\widetilde{u}_{\varepsilon}}{\varepsilon^{2}}\right\|_{L_{T}^{2}\left(L^{2}(\Omega)\right)} \leq \|\left.\frac{u_{\varepsilon}}{\varepsilon}\right|_{L_{T}^{2}\left(H_{0}^{1}\left(\Omega_{\varepsilon}\right)\right)}$, we deduce the existence of some $u \in L_{T}^{2}\left(L^{2}(\Omega)\right)$ and of a subsequence $\frac{\widetilde{u}_{\varepsilon}}{\varepsilon^{2}}$ which converges weakly to $u$.

Finally, from the bound we have on $\widehat{p}_{\varepsilon}$, we can deduce the existence of some $p \in L_{T}^{2}\left(H^{1}(\Omega)\right)$ such that $p_{\varepsilon}$ converges weakly to $p$ in $L_{T}^{2}\left(L^{2}(\Omega)\right)$. However, we can not deduce strong convergence since we do not have compactness in time. To recover some compactness in time, we will use the conservation of mass equation which provides some compactness in time for $\rho_{\varepsilon}$. We start by prolonging $\rho_{\varepsilon}$ in a suitable way.

Lemma 2.3. the extension $\widetilde{\rho}_{\varepsilon}$ satisfies the following equation

$$
\varepsilon^{2} \partial_{t} \widetilde{\rho}_{\varepsilon}+\operatorname{div}\left(\widetilde{\rho}_{\varepsilon} \widetilde{u}_{\varepsilon}\right)=0 \text { in } \Omega .
$$

Proof. In this proof, $\varepsilon$ is supposed to be fixed. For any $\delta$ small enough, we consider $\phi_{\delta} \in \mathcal{D}\left(\Omega_{\varepsilon}\right)$ such that

$$
\left\{\begin{array}{l}
0 \leq \phi_{\delta} \leq 1, \quad \text { in } \Omega_{\varepsilon}, \quad \phi_{\delta}=1 \text { if } d(x) \geq \delta \\
\phi_{\delta}=0 \text { if } d(x) \leq \frac{\delta}{2}, \quad\left|\nabla \phi_{\delta}\right| \leq \frac{C}{\delta} \quad \text { in } \Omega_{\varepsilon}
\end{array}\right.
$$

where $d=d\left(x, \partial \Omega_{\varepsilon}\right)$ and $C$ is a constant independent of $\delta$ (but depending on $\varepsilon$ ). We also recall Hardy inequality which implies that for all $\varepsilon, \frac{u_{\varepsilon}}{d} \in L^{2}\left((0, T) \times \Omega_{\varepsilon}\right)$ since $u_{\varepsilon} \in L^{2}\left(0, T ; H_{0}^{1}\left(\Omega_{\varepsilon}\right)\right)$. Now, for all $\psi \in \mathcal{D}((0, T) \times \Omega)$, 
we have

$$
\begin{aligned}
\int_{0}^{T} \int_{\Omega} \tilde{\rho}_{\varepsilon} \widetilde{u}_{\varepsilon} \cdot \nabla \psi & =\int_{0}^{T} \int_{\Omega_{\varepsilon}} \rho_{\varepsilon} u_{\varepsilon} \cdot \nabla \psi=\lim _{\delta \rightarrow 0} \int_{0}^{T} \int_{\Omega_{\varepsilon}} \rho_{\varepsilon} u_{\varepsilon} \cdot \nabla\left(\psi \phi_{\delta}\right) \\
& =\lim _{\delta \rightarrow 0} \int_{0}^{T} \int_{\Omega_{\varepsilon}} \rho_{\varepsilon} \partial_{t} \psi \phi_{\delta}=\int_{0}^{T} \int_{\Omega} \tilde{\rho}_{\varepsilon} \partial_{t} \psi
\end{aligned}
$$

where we have used that

$$
\begin{aligned}
\int_{0}^{T} \int_{\Omega_{\varepsilon}}\left|\rho_{\varepsilon} u_{\varepsilon}\right|\left(1-\phi_{\delta}+\left|\nabla \phi_{\delta}\right|\right) & =\int_{0}^{T} \int_{\Omega_{\varepsilon} \cap\{d \leq \delta\}}\left|\rho_{\varepsilon} u_{\varepsilon}\right|\left(1-\phi_{\delta}+\left|\nabla \phi_{\delta}\right|\right) \\
& \leq \int_{0}^{T} \int_{\Omega_{\varepsilon} \cap\{d \leq \delta\}}\left|\rho_{\varepsilon} u_{\varepsilon}\right|\left(1+\frac{2}{d}\right) \underset{\delta \rightarrow 0}{\longrightarrow} 0
\end{aligned}
$$

to pass from the first line to the second one.

Using that $\widetilde{\rho}_{\varepsilon}$ is bounded in $L^{2}((0, T) \times \Omega)$ and that $\frac{\widetilde{u}_{\varepsilon}}{\varepsilon^{2}}$ is also bounded in $L^{2}((0, T) \times \Omega)$, we deduce that $\partial_{t} \widetilde{\rho}_{\varepsilon}$ is bounded in $L^{1}\left(0, T ; W^{-1,1}(\Omega)\right)$. We can now use Lemma 5.1 of [14] to pass to the limit in the product $\widetilde{\rho}_{\varepsilon} \widehat{p}_{\varepsilon}$ and deduce that $\widetilde{\rho}_{\varepsilon} \widehat{p}_{\varepsilon}=\left(\widetilde{\rho}_{\varepsilon}\right)^{\gamma+1}=\widetilde{\rho_{\varepsilon}^{\gamma+1}}$ converges weakly to $\theta \rho p$. Then, using Lemma 1.3, we deduce that $\widehat{\rho_{\varepsilon}^{\gamma+1}}$ converges weakly to $\rho p$. Now, by Jensen inequality, we have

$$
\widehat{\rho_{\varepsilon}^{\gamma+1}} \geq\left(\widehat{\rho_{\varepsilon}^{\gamma}}\right)^{\frac{\gamma+1}{\gamma}}=\left(\widehat{p_{\varepsilon}}\right)^{\frac{\gamma+1}{\gamma}} .
$$

Passing to the weak limit, we get

$$
\rho p \geq \mathrm{w}-\lim \left(\widehat{p_{\varepsilon}}\right)^{\frac{\gamma+1}{\gamma}} \geq p^{\frac{\gamma+1}{\gamma}}
$$

from which we deduce that

$$
\rho \geq p^{\frac{1}{\gamma}}
$$

Next, we define $\left.\chi_{\varepsilon}=\widehat{\left(\rho_{\varepsilon}^{\gamma}\right.}\right)^{\frac{1}{\gamma}}$ and extracting a subsequence, if necessary, $\chi_{\varepsilon}$ converges weakly to some $\chi$. Using that $\gamma \geq 1$, we deduce that $\chi^{\gamma} \leq p$. Then, by Jensen inequality, we have

$$
\widehat{\rho_{\varepsilon}} \leq\left(\widehat{\rho_{\varepsilon}^{\gamma}}\right)^{\frac{1}{\gamma}}=\chi_{\varepsilon}
$$

Passing to the weak limit, we get that $\rho \leq \chi$. Putting all the above inequalities together, we get

$$
\rho \leq \chi \leq p^{\frac{1}{\gamma}} \leq \rho
$$

and hence $\rho=\chi=p^{\frac{1}{\gamma}}$. Next, we use that $\chi_{\varepsilon}^{\gamma+1}$ converges weakly to $\chi^{\gamma+1}$ to deduce the strong convergence of $\chi_{\varepsilon}$ towards $\chi$ in $L^{\gamma}((0, T) \times \Omega)$. Using the $L^{2 \gamma}$ bound for $\chi_{\varepsilon}$, we deduce that $\widehat{p}_{\varepsilon}$ converges strongly to $p$ in all the $L^{r}((0, T) \times \Omega)$ where $r<2$. On the other hand, we know that $\widehat{\rho}_{\varepsilon}$ converges weakly to $\rho=\chi$ and that

$$
\rho^{\gamma+1} \leq \mathrm{w}-\lim \left(\widehat{\rho_{\varepsilon}}\right)^{\gamma+1} \leq \mathrm{w}-\lim \widehat{\rho_{\varepsilon}^{\gamma+1}}=\rho^{\gamma+1}
$$

from which we deduce the equality in $(48)$ and the fact that $\widehat{\rho}_{\varepsilon}$ converges strongly to $\rho$ in $L^{2}((0, T) \times \Omega)$. 
Now, we want to compute the weak limit of $\widetilde{u}_{\varepsilon}$. Using that $P_{\varepsilon}=\widehat{p_{\varepsilon}^{\gamma}} \in L^{2}\left(H^{1}\right)+\varepsilon L^{2}\left(L^{2}\right)$, we will make spatial regularization of $P_{\varepsilon}$ : take $\chi \in C_{0}^{\infty}\left(\mathbb{R}^{N}\right)$ such that $\chi \geq 0, \int_{\mathbb{R}^{N}} \chi=1$ and denote for all $\eta \in(0,1)$ by $\chi_{\eta}(x)=\frac{1}{\eta^{N}} \chi\left(\frac{x}{\eta}\right)$. We then define for all $1>\eta>0, P_{\varepsilon, \eta}=P_{\varepsilon}^{*} \chi_{x} \chi_{\eta}+\eta$ where we have prolonged $P_{\varepsilon}$ by 0 outside the domain $\Omega$. Then, using that $P_{\varepsilon} \in L^{2}\left(H^{1}\right)+\varepsilon L^{2}\left(L^{2}\right)$ we deduce that

$$
\left\|P_{\varepsilon}-P_{\varepsilon, \eta}\right\|_{L^{2}\left(L^{2}\right)} \leq C(\varepsilon+\eta) .
$$

We also denote $\rho_{\varepsilon, \eta}=\left(P_{\varepsilon, \eta}\right)^{\frac{1}{\gamma}}$. It is easy to see that for all $1>\eta>0, \rho_{\varepsilon, \eta} \in L^{2}\left(H^{1}\right)$ and that

$$
\left\|\rho_{\varepsilon, \eta}\right\|_{L^{2}\left(H^{1}\right)} \leq C \eta^{\frac{1}{\gamma}-1} .
$$

Using the system (26), we define the functions $\left(v_{k}^{\varepsilon}, q_{k}^{\varepsilon}\right) \in H^{1}(\Omega) \times L^{2}\left(\Omega_{\varepsilon}\right)$ by

$$
\left\{\begin{array}{l}
v_{k}^{\varepsilon}=v_{k}\left(\frac{x}{\varepsilon}\right), \\
q_{k}^{\varepsilon}=q_{k}\left(\frac{x}{\varepsilon}\right)
\end{array}\right.
$$

extended in $\Omega$ and in $\Omega_{\varepsilon}$ by $\varepsilon \mathcal{Y}$-periodicity. Hence, we have the following estimates

$$
\begin{gathered}
\left\|q_{k}^{\varepsilon}\right\|_{L^{\infty}\left(\Omega_{\varepsilon}\right)} \leq C, \quad\left\|\varepsilon \nabla q_{k}^{\varepsilon}\right\|_{L^{\infty}\left(\Omega_{\varepsilon}\right)} \leq C, \\
\left\|v_{k}^{\varepsilon}\right\|_{L^{\infty}(\Omega)} \leq C, \quad\left\|\varepsilon \nabla v_{k}^{\varepsilon}\right\|_{L^{\infty}(\Omega)} \leq C .
\end{gathered}
$$

For all $\phi \in \mathcal{D}((0, T) \times \Omega)$, we take $\rho_{\varepsilon, \eta} v_{k}^{\varepsilon} \phi$ as a test function in (27). For $\varepsilon$ small enough, we know that $\operatorname{Supp}(\phi) \subset U_{k \in K_{\varepsilon}} \varepsilon \mathcal{Y}^{k}$ and

$$
\int_{0}^{T} \int_{\Omega_{\varepsilon}} \mu \nabla u_{\varepsilon} \cdot \nabla\left(\rho_{\varepsilon, \eta} v_{k}^{\varepsilon} \phi\right)=\int_{0}^{T} \int_{\Omega}\left(\rho_{\varepsilon, \eta} v_{k}^{\varepsilon} \phi\right)\left(\rho_{\varepsilon} f+g\right)+\int_{0}^{T} \int_{\Omega} \operatorname{div}\left(\rho_{\varepsilon, \eta} v_{k}^{\varepsilon} \phi\right)\left(\widehat{\rho_{\varepsilon}^{\gamma}}-\xi \operatorname{div} u_{\varepsilon}\right) .
$$

In the right hand side, we have replaced the integrations over $\Omega_{\varepsilon}$ by integration over $\Omega$ since $\phi v_{k}^{\varepsilon}$ and $\phi \operatorname{div}\left(v_{k}^{\varepsilon}\right)$ vanish on $\Omega-\Omega_{\varepsilon}$.

Now for all $\eta>0$, we have

$$
\left.\int_{0}^{T} \int_{\Omega} \operatorname{div}\left(\phi \rho_{\varepsilon, \eta} v_{k}^{\varepsilon}\right) \widehat{\left(\rho_{\varepsilon}^{\gamma}\right.}-P_{\varepsilon, \eta}\right)=\int_{0}^{T} \int_{\Omega} \nabla \rho_{\varepsilon, \eta} \cdot v_{k}^{\varepsilon} \phi\left(P_{\varepsilon}-P_{\varepsilon, \eta}\right)+\int_{0}^{T} \int_{\Omega} \rho_{\varepsilon, \eta} v_{k}^{\varepsilon} \cdot \nabla \phi\left(P_{\varepsilon}-P_{\varepsilon, \eta}\right)
$$

and hence

$$
\left|\int_{0}^{T} \int_{\Omega} \operatorname{div}\left(\phi \rho_{\varepsilon, \eta} v_{k}^{\varepsilon}\right)\left(P_{\varepsilon}-P_{\varepsilon, \eta}\right)\right| \leq C\left(\eta^{\frac{1}{\gamma}-1}+1\right)(\varepsilon+\eta)=\alpha(\eta, \varepsilon)
$$

where here and below, $\alpha(\eta, \varepsilon)$ denotes any function such that

$$
\lim _{\eta \rightarrow 0} \lim _{\varepsilon \rightarrow 0} \alpha(\eta, \varepsilon)=0
$$

and $\alpha(\eta)$ any function such that $\lim _{\eta \rightarrow 0} \alpha(\eta)=0$. With these notations, we have

$$
\int_{0}^{T} \int_{\Omega} \mu \nabla \widetilde{u}_{\varepsilon} \cdot \nabla\left(\rho_{\varepsilon, \eta} v_{k}^{\varepsilon} \phi\right)=\int_{0}^{T} \int_{\Omega} v_{k}^{\varepsilon} \phi\left(\rho_{\varepsilon, \eta}^{2} f+\rho_{\varepsilon, \eta} g-\frac{\gamma}{\gamma+1} \nabla \rho_{\varepsilon, \eta}^{\gamma+1}\right)+\alpha(\eta, \varepsilon) .
$$


On the other hand using that $-\varepsilon^{2} \Delta v_{k}^{\varepsilon}+\varepsilon \nabla q_{k}^{\varepsilon}=e_{k}$ in $\Omega_{\varepsilon}$ and taking $\rho_{\varepsilon, \eta} \frac{u_{\varepsilon}}{\varepsilon^{2}} \phi$ as a test function, we get

$$
\int_{0}^{T} \int_{\Omega_{\varepsilon}} \nabla v_{k}^{\varepsilon} \cdot \nabla\left(\rho_{\varepsilon, \eta} u_{\varepsilon} \phi\right)+\frac{1}{\varepsilon} \int_{0}^{T} \int_{\Omega_{\varepsilon}} \rho_{\varepsilon, \eta} u_{\varepsilon} \cdot \nabla q_{k}^{\varepsilon} \phi=\int_{0}^{T} \int_{\Omega_{\varepsilon}} \rho_{\varepsilon, \eta} \frac{u_{\varepsilon} e_{k}}{\varepsilon^{2}} \phi
$$

The second term on the left hand side can be estimated as follows

$$
\begin{aligned}
\frac{1}{\varepsilon} \int_{0}^{T} \int_{\Omega_{\varepsilon}} \rho_{\varepsilon, \eta} u_{\varepsilon} \cdot \nabla q_{k}^{\varepsilon} \phi= & \int_{0}^{T} \int_{\Omega_{\varepsilon}} \rho_{\varepsilon, \eta} \frac{u_{\varepsilon}}{\varepsilon^{2}} \varepsilon \nabla q_{k}^{\varepsilon} \phi \\
= & \int_{0}^{T} \int_{\Omega_{\varepsilon}} \rho_{\varepsilon} \frac{u_{\varepsilon}}{\varepsilon^{2}} \varepsilon \nabla q_{k}^{\varepsilon} \phi+\alpha(\eta, \varepsilon) \\
= & -\int_{0}^{T} \int_{\Omega_{\varepsilon}} \operatorname{div}\left(\rho_{\varepsilon} \frac{u_{\varepsilon}}{\varepsilon^{2}}\right) \varepsilon q_{k}^{\varepsilon} \phi \\
& -\int_{0}^{T} \int_{\Omega_{\varepsilon}} \rho_{\varepsilon} \frac{u_{\varepsilon}}{\varepsilon^{2}} \varepsilon q_{k}^{\varepsilon} \nabla \phi+\alpha(\eta, \varepsilon) \\
= & \alpha(\eta, \varepsilon)
\end{aligned}
$$

where we have used that

$$
\int_{0}^{T} \int_{\Omega_{\varepsilon}} \operatorname{div}\left(\rho_{\varepsilon} \frac{u_{\varepsilon}}{\varepsilon^{2}}\right) \varepsilon q_{k}^{\varepsilon} \phi=\int_{0}^{T} \int_{\Omega_{\varepsilon}} \rho_{\varepsilon} \varepsilon q_{k}^{\varepsilon} \partial_{t} \phi \rightarrow 0 \text { as } \varepsilon \text { goes to } 0 .
$$

Comparing (53) and (55), we get

$$
\int_{0}^{T} \int_{\Omega}\left(\nabla \tilde{u}_{\varepsilon} \cdot \nabla\left(\rho_{\varepsilon, \eta} v_{k}^{\varepsilon} \phi\right)-\nabla v_{k}^{\varepsilon} \cdot \nabla\left(\rho_{\varepsilon, \eta} \tilde{u}_{\varepsilon} \phi\right)\right)=\varepsilon \int_{0}^{T} \int_{\Omega}\left[\nabla \rho_{\varepsilon, \eta} \phi+\rho_{\varepsilon, \eta} \nabla \phi\right]\left(\frac{\tilde{u}_{\varepsilon}}{\varepsilon^{2}} \varepsilon \nabla v_{k}^{\varepsilon}-\frac{\nabla \tilde{u}_{\varepsilon}}{\varepsilon} v_{k}^{\varepsilon}\right)
$$

which goes to 0 when $\varepsilon$ goes to 0 for all $\eta>0$. For all $\eta>0$, we denote $\bar{\rho}_{\eta}$ the strong limit in $L^{2}$ of $\rho_{\varepsilon, \eta}$ when $\varepsilon$ goes to 0 . Multiplying (55) by $\mu$, taking the difference with (54) and passing to the limit in $\varepsilon$, we get

$$
\int_{0}^{T} \int_{\Omega} \bar{A} e_{k} \cdot\left(\bar{\rho}_{\eta}^{2} f+\bar{\rho}_{\eta} g-\frac{\gamma}{\gamma+1} \nabla \bar{\rho}_{\eta}^{\gamma+1}\right) \phi=\mu \int_{0}^{T} \int_{\Omega} \bar{\rho}_{\eta} u e_{k} \phi \quad+\alpha(\eta)
$$

where we have used that $v_{k}^{\varepsilon}$ converges weakly in $L^{2}(\Omega)$ to the constant vector $\int_{\mathcal{Y}_{f}} v_{k}(y) \mathrm{d} y=\bar{A} e_{k}$, that $\left(\rho_{\varepsilon, \eta}^{2} f+\rho_{\varepsilon, \eta} g-\nabla \rho_{\varepsilon, \eta}^{\gamma+1}\right)$ converges strongly in $L^{1}((0, T) \times \Omega)$ to $\left(\bar{\rho}_{\eta}^{2} f+\bar{\rho}_{\eta} g-\nabla \bar{\rho}_{\eta}^{\gamma+1}\right)$ and that $\rho_{\varepsilon, \eta}$ converges strongly in $L^{2}((0, T) \times \Omega)$ to $\bar{\rho}_{\eta}$. Then, passing to the limit in $\eta$, we deduce that

$$
\int_{0}^{T} \int_{\Omega} \bar{A} e_{k} \cdot\left(\rho^{2} f+\rho g-\frac{\gamma}{\gamma+1} \nabla \rho^{\gamma+1}\right)=\mu \int_{0}^{T} \int_{\Omega} \rho u e_{k} \phi .
$$

Hence, we infer that

$$
\rho u=\frac{1}{\mu} \bar{A}\left(\rho^{2} f+\rho g-\frac{\gamma}{\gamma+1} \nabla \rho^{\gamma+1}\right)
$$

from which we also deduce that

$$
u=\frac{1}{\mu} \bar{A}\left(\rho f+g-\nabla \rho^{\gamma}\right) \quad \text { on the set } \quad\{\rho>0\} .
$$


Finally, we have to pass to the limit in the conservation of mass to recover the equation satisfied by $\rho$. First, we notice that (42) can be rewritten as

$$
\partial_{t} \widetilde{\rho}_{\varepsilon}+\operatorname{div}\left(\widehat{\rho}_{\varepsilon} \frac{\widetilde{u}_{\varepsilon}}{\varepsilon^{2}}\right)=0 \quad \text { in } \quad \Omega
$$

The advantage of replacing $\widetilde{\rho}_{\varepsilon}$ by $\widehat{\rho}_{\varepsilon}$ lays in the fact that the latter converges strongly while the former converges weakly. Passing to the limit in (60), we deduce the first equation in the limiting system (28). To recover the boundary condition as well as the initial data, we have to use some weak formulation. If we also denote $\widetilde{\rho}_{\varepsilon}$, $\widetilde{u}_{\varepsilon}$ the extensions by 0 of $u_{\varepsilon}$ and $\rho_{\varepsilon}$ to $\mathbb{R}^{N}$, then an adaptation of Lemma 2.3 also implies that (42) holds in $\mathbb{R}^{N}$. As above, we can then write

$$
\partial_{t} \widetilde{\rho}_{\varepsilon}+\operatorname{div}\left(\widehat{\rho}_{\varepsilon} \frac{\widetilde{u}_{\varepsilon}}{\varepsilon^{2}}\right)=0 \quad \text { in } \quad \mathbb{R}^{N}
$$

For all $\phi \in \mathcal{D}\left((-1, T) \times \mathbb{R}^{N}\right)$, we have

$$
-\int_{0}^{T} \int_{\mathbb{R}^{N}} \widetilde{\rho}_{\varepsilon} \partial_{t} \phi-\int_{0}^{T} \int_{\mathbb{R}^{N}} \widehat{\rho}_{\varepsilon} \frac{\widetilde{u}_{\varepsilon}}{\varepsilon^{2}} \cdot \nabla \phi=\int_{\mathbb{R}^{N}} \widetilde{\rho}_{\varepsilon}^{0} \phi(t=0) .
$$

The three integration over $\mathbb{R}^{N}$ appearing in the above identity can be be replaced by integration over $\Omega$. Passing to the limit we deduce

$$
-\int_{0}^{T} \int_{\Omega} \theta \rho \partial_{t} \phi-\int_{0}^{T} \int_{\Omega} \frac{1}{\mu} \bar{A}\left(\rho^{2} f+\rho g-\frac{\gamma}{\gamma+1} \nabla \rho^{\gamma+1}\right) \cdot \nabla \phi=\int_{\Omega} \theta \rho^{0} \phi(t=0)
$$

for all $\phi \in C^{\infty}([0, T) \times \bar{\Omega})$. Hence, we recover the boundary condition and the initial data in (28).

\section{Compressible Navier-Stokes system}

Here, we restrict ourselves to the case $N=2$ or $N=3$ and we consider the full system

$$
\left\{\begin{array}{l}
\varepsilon^{2} \partial_{t} \rho_{\epsilon}+\operatorname{div}\left(\rho_{\epsilon} u_{\epsilon}\right)=0 \\
\varepsilon^{2} \partial_{t}\left(\rho_{\varepsilon} u_{\varepsilon}\right)+\operatorname{div}\left(\rho_{\epsilon} u_{\epsilon} \otimes u_{\epsilon}\right)-\mu \Delta u_{\epsilon}-\xi \nabla \operatorname{div} u_{\epsilon}+\nabla \rho_{\epsilon}^{\gamma}=\rho_{\varepsilon} f+g
\end{array}\right.
$$

complemented with the boundary condition $u_{\varepsilon}=0$ on $\partial \Omega_{\varepsilon}$ and the initial conditions $\rho_{\varepsilon}(t=0)=\rho_{\varepsilon 0}$ and $\rho_{\varepsilon} u_{\varepsilon}(t=0)=m_{\varepsilon 0}$. We want to prove the same convergence result as in Theorem 2.1. We will require that $\gamma \geq N$ (and for $N=2$ that $\gamma>2$ ) even though the existence results of global weak solutions available in the literature only requires $\gamma>\frac{N}{2}$ (see [14] and [9]). As in the previous section, we assume that the initial data is such that $\rho_{\varepsilon 0} \in L^{1} \cap L^{\gamma}\left(\Omega_{\varepsilon}\right), m_{\varepsilon 0} \in L^{\frac{2 \gamma}{\gamma+1}}\left(\Omega_{\varepsilon}\right)$ and $m_{\varepsilon 0}=0$ a.e on the set $\left\{\rho_{\varepsilon 0}=0\right\}, \rho_{\varepsilon 0}\left|u_{\varepsilon 0}\right|^{2} \in L^{1}\left(\Omega_{\varepsilon}\right)$ where we denote $u_{\varepsilon 0}=\frac{m_{\varepsilon 0}}{\rho_{\varepsilon 0}}$ on $\left\{\rho_{\varepsilon 0}>0\right\}$ and $u_{\varepsilon 0}=0$ on $\left\{\rho_{\varepsilon 0}=0\right\}$. Moreover, we assume that $\widehat{\rho}_{\varepsilon 0}$ converges weakly to $\rho_{0}$ in $L^{\gamma}(\Omega)$.

We consider a sequence of weak solutions $\left(\rho_{\varepsilon}, u_{\varepsilon}\right)$ of the compressible Navier-Stokes system $(63)$ such that $\rho_{\varepsilon} \in C\left([0, T) ; L^{1}\left(\Omega_{\varepsilon}\right)\right) \cap L^{\infty}\left(0, T ; L^{\gamma}\left(\Omega_{\varepsilon}\right)\right) \cap L^{\gamma+1}\left((0, T) \times \Omega_{\varepsilon}\right)$. Moreover, $u_{\varepsilon}$ is such that $\frac{u_{\varepsilon}}{\varepsilon} \in L^{2}\left(0, T ; H_{0}^{1}\left(\Omega_{\varepsilon}\right)\right)$ and $\frac{u_{\varepsilon}}{\varepsilon^{2}} \in L^{2}\left((0, T) \times \Omega_{\varepsilon}\right)$. 
Theorem 3.1. Under the above assumptions,

$$
\begin{aligned}
& \widetilde{\rho}_{\varepsilon} \quad \rightarrow \quad \theta \rho \quad \text { weakly in } L_{T}^{r}\left(L^{\gamma}(\Omega)\right) \cap L^{\gamma+1}((0, T) \times \Omega), \\
& \widehat{\rho}_{\varepsilon} \rightarrow \quad \rho \quad \text { strongly in } \quad L_{T}^{r}\left(L^{\gamma}(\Omega)\right) \cap L^{\gamma+1}((0, T) \times \Omega), \\
& \frac{\widetilde{u}_{\varepsilon}}{\varepsilon^{2}} \quad \rightarrow \quad u \quad \text { weakly in } L_{T}^{2}\left(L^{2}(\Omega)\right)
\end{aligned}
$$

for all $r<\infty$ where $\rho \in L^{2 \gamma}((0, T) \times \Omega)$, $\rho^{\gamma} \in L_{T}^{2}\left(H^{1}(\Omega)\right)$ and $\rho$ is the solution of the same limit system (28) and $u$ is given by the same formula (29).

\subsection{A priori estimates}

Here we want to explain the changes we have to make in the a priori estimates. As above, we can deduce the conservation of the total mass from the continuity equations. The energy estimate (31) should be replaced by

$$
\varepsilon^{2} \int_{\Omega_{\varepsilon}} \frac{\rho_{\varepsilon}^{\gamma}(t)}{\gamma-1}+\frac{\rho_{\varepsilon}(t)\left|u_{\varepsilon}^{2}(t)\right|}{2}+\int_{0}^{t} \int_{\Omega_{\varepsilon}} \mu\left|\nabla u_{\varepsilon}\right|^{2}+\xi\left(\operatorname{div} u_{\varepsilon}\right)^{2}=\varepsilon^{2} \int_{\Omega_{\varepsilon}} \frac{\rho_{\varepsilon 0}^{\gamma}}{\gamma-1}+\frac{\rho_{\varepsilon 0}\left|u_{\varepsilon 0}^{2}\right|}{2}+\int_{0}^{t} \int_{\Omega_{\varepsilon}}\left(\rho_{\varepsilon} f+g\right) \cdot u_{\varepsilon} .
$$

As in the previous section, we can deduce from (64) that for all $T$ there exists a constant $C_{T}$ such that

$$
\sup _{0 \leq t \leq T} \int_{\Omega_{\varepsilon}} \rho_{\varepsilon}^{\gamma}(t)+\frac{\rho_{\varepsilon}(t)\left|u_{\varepsilon}^{2}(t)\right|}{2}+\int_{0}^{T} \int_{\Omega_{\varepsilon}}\left|\nabla \frac{u_{\varepsilon}}{\varepsilon}\right|^{2} \leq C_{T}
$$

We can also deduce some bound on $\widehat{p}_{\varepsilon}$, however it will not be as good as for the semi-stationary system due to the presence of a time derivative. Let us start by some space-time integrability of the pressure.

Let $v=\mathcal{B}\left(\rho_{\varepsilon}-\frac{1}{\left|\Omega_{\varepsilon}\right|} \int_{\Omega_{\varepsilon}} \rho_{\varepsilon}\right)$ where $\mathcal{B}$ was defined in $(10)$ then

$$
\begin{gathered}
\|v\|_{L^{2}\left(\Omega_{\varepsilon}\right)} \leq C \varepsilon\|\nabla v\|_{L^{2}\left(\Omega_{\varepsilon}\right)} \leq C\left\|\rho_{\varepsilon}\right\|_{L^{2}\left(\Omega_{\varepsilon}\right)} \\
\|v\|_{L^{\gamma}\left(\Omega_{\varepsilon}\right)} \leq C \varepsilon\|\nabla v\|_{L^{\gamma}\left(\Omega_{\varepsilon}\right)} \leq C\left\|\rho_{\varepsilon}\right\|_{L^{\gamma}\left(\Omega_{\varepsilon}\right)} .
\end{gathered}
$$

Multiplying the second equation of (27) by $v$ and integrating by parts, we get at least formally (we drop the $\varepsilon$ )

$$
\begin{aligned}
\int_{0}^{T} \int_{\Omega_{\varepsilon}} \rho^{\gamma+1}= & \int_{0}^{T} \int_{\Omega_{\varepsilon}} \rho^{\gamma} \times \int_{\Omega_{\varepsilon}} \rho+\int_{0}^{T} \int_{\Omega_{\varepsilon}} \mu \nabla u \cdot \nabla v+\xi(\operatorname{div} u)(\operatorname{div} v) \\
& -\int_{0}^{T} \int_{\Omega_{\varepsilon}}(\rho f+g) \cdot v-\int_{0}^{T} \int_{\Omega_{\varepsilon}} \varepsilon^{2} \rho u \partial_{t} v-\int_{0}^{T} \int_{\Omega_{\varepsilon}} \rho u \otimes u: \nabla v \\
& +\varepsilon^{2} \int_{\Omega_{\varepsilon}} m_{\varepsilon 0} v(0)-\rho u \cdot v(T) \\
= & \sum_{i=1}^{6} I_{i} .
\end{aligned}
$$

We have to estimate each one of the six terms appearing in (68). First, we have

$$
\left|I_{1}\right| \leq C_{T} T \int_{\Omega_{\varepsilon}} \rho_{\varepsilon 0} \leq C_{T}
$$


The second term is such that

$$
\left|I_{2}\right| \leq C_{T} \varepsilon|| \nabla v \|_{L_{T}^{2}\left(\Omega_{\varepsilon}\right)} \leq C_{T}
$$

For the third term, we have

$$
\left|I_{3}\right| \leq\left(\left\|\left.f\right|_{L^{\infty}}\right\| \rho\left\|_{L_{T}^{2}\left(\Omega_{\varepsilon}\right)}+\right\| g \|_{L_{T}^{2}\left(\Omega_{\varepsilon}\right)}\right)\|v\|_{L_{T}^{2}\left(\Omega_{\varepsilon}\right)} \leq C_{T} .
$$

The fourth term is the most technical and requires some spatial regularization of $v$ (see for instance [9]). Taking the time derivative of $v$, we get $\partial_{t} v=\mathcal{B}\left(\partial_{t} \rho_{\varepsilon}\right)=-\mathcal{B}\left(\operatorname{div} \rho_{\varepsilon} \frac{u_{\varepsilon}}{\varepsilon^{2}}\right)$. Hence, using (13), we get

$$
\left\|\partial_{t} v\right\|_{L_{T}^{2}\left(\Omega_{\varepsilon}\right)} \leq \frac{C}{\varepsilon^{2}}\left\|\rho_{\varepsilon} u_{\varepsilon}\right\|_{L_{T}^{2}\left(\Omega_{\varepsilon}\right)} .
$$

This last inequality requires some explanations, since we do not know if $\operatorname{div} \rho_{\varepsilon} u_{\varepsilon}$ is in any $L^{p}$ space and it is not clear whether $\rho_{\varepsilon} u_{\varepsilon} . n=0$ on $\partial \Omega_{\varepsilon}$. These two difficulties can be overcome if we are willing to use spaces with negative regularity in time. Another way is to regularize $\rho_{\varepsilon}$ in space. We will give a sketch of this regularization after we perform the estimate of the last three terms. From (69), we deduce (at least for $N \geq 3$ ) that

$$
\left|I_{4}\right| \leq C|| \rho_{\varepsilon} u_{\varepsilon}\left\|_{L_{T}^{2}\left(\Omega_{\varepsilon}\right)}^{2} \leq C\right\| \rho_{\varepsilon}\left\|_{L_{T}^{\infty}\left(L^{N}\left(\Omega_{\varepsilon}\right)\right)}^{2}\right\| u_{\varepsilon} \|_{L_{T}^{2}\left(L^{\frac{2 N}{N-2}}\left(\Omega_{\varepsilon}\right)\right)}^{2} \leq C_{T} \varepsilon^{2} .
$$

For $N=2$, we use that $\gamma>2$ and hence

$$
\left|I_{4}\right| \leq C|| \rho_{\varepsilon} u_{\varepsilon}\left\|_{L_{T}^{2}\left(\Omega_{\varepsilon}\right)}^{2} \leq C\right\| \rho_{\varepsilon}\left\|_{L_{T}^{\infty}\left(L^{\gamma}\left(\Omega_{\varepsilon}\right)\right)}^{2} \mid\right\| u_{\varepsilon} \|_{L_{T}^{2}\left(L^{\frac{2 \gamma}{\gamma-2}}\left(\Omega_{\varepsilon}\right)\right)}^{2} \leq C_{T} \varepsilon^{2} .
$$

For $I_{5}$, we have

$$
\left|I_{5}\right| \leq C|| \rho\left\|_{L_{T}^{\infty}\left(L^{\gamma}\left(\Omega_{\varepsilon}\right)\right)}\right\| u_{\varepsilon}\left\|_{L_{T}^{2}\left(L^{\frac{2 \gamma}{\gamma-2}}\right)}^{2}\right\| \nabla v \|_{L_{T}^{\infty}\left(L^{\gamma}\left(\Omega_{\varepsilon}\right)\right)} \leq C_{T} \varepsilon .
$$

Finally, $I_{6}$ is estimated in the following way

$$
\left|I_{6}\right| \leq C \varepsilon\left\|\rho_{\varepsilon} \frac{\left|u_{\varepsilon}\right|^{2}}{2}\right\|_{L_{T}^{\infty}\left(L^{1}\left(\Omega_{\varepsilon}\right)\right)}^{\frac{1}{2}}\left\|\rho_{\varepsilon}\right\|_{L_{T}^{\infty}\left(L^{\gamma}\left(\Omega_{\varepsilon}\right)\right)}^{\frac{1}{2}}\|\varepsilon v\|_{L_{T}^{\infty}\left(L^{\left.\frac{2 \gamma}{\gamma-1}\right)}\right.} \leq C_{T} \varepsilon .
$$

The a priori estimate is hence proved. Let us now explain how the difficulty related to $I_{4}$ can be solved. We take as above $\chi \in C_{0}^{\infty}\left(\mathbb{R}^{N}\right)$ such that $\chi \geq 0, \int_{\mathbb{R}^{N}} \chi=1$ and denote for all $\delta \in(0,1)$ by $\chi_{\delta}(x)=\frac{1}{\delta^{N}} \chi\left(\frac{x}{\delta}\right)$. Next, we denote $\rho_{\varepsilon, \delta}=\widetilde{\rho}_{\varepsilon}{ }_{x}^{*} \chi_{\delta}$ where here $\widetilde{\rho}_{\varepsilon}$ denotes the extension of $\rho_{\varepsilon}$ to the whole of $\mathbb{R}^{N}$. Using (42) written in $\mathbb{R}^{N}$, we deduce the following relation

$$
\varepsilon^{2} \partial_{t} \widetilde{\rho}_{\varepsilon, \delta}+\operatorname{div}\left(\widetilde{\rho}_{\varepsilon, \delta} \widetilde{u}_{\varepsilon}\right)=r_{\varepsilon, \delta} \quad \text { in } \quad \mathbb{R}^{n}
$$

where for all fixed $\varepsilon, r_{\varepsilon, \delta}$ goes to 0 in $L_{T}^{2}\left(L^{\frac{2 \gamma}{2+\gamma}}(\Omega)\right.$ ) (see Lem. 2.3 of [13]). Next, instead of using $v$ as a test function, we will use $v^{\delta}=\mathcal{B}\left(\rho_{\varepsilon, \delta}-\frac{1}{\left|\Omega_{\varepsilon}\right|} \int_{\Omega_{\varepsilon}} \rho_{\varepsilon, \delta}\right)$. The estimates (66) and (67) still hold with bounds independent of $\delta$. Moreover, (68) can now be rewritten as

$$
\int_{0}^{T} \int_{\Omega_{\varepsilon}} \rho^{\gamma} \rho_{\varepsilon, \delta}=\sum_{i=1}^{6} I_{i}^{\delta}
$$


We can estimate each one of the terms $I_{i}^{\delta}$ as above and independently of $\delta$. Let us just explain it on $I_{4}^{\delta}$. We have $\left.\partial_{t} v^{\delta}=\mathcal{B}\left(\partial_{t} \rho_{\varepsilon, \delta}-\frac{1}{\Omega_{\varepsilon}} \partial_{t} \int_{\Omega_{\varepsilon}} \rho_{\varepsilon}\right)\right)$ and hence

$$
\partial_{t} v^{\delta}=-\mathcal{B}\left(\operatorname{div} \rho_{\varepsilon, \delta} \frac{u_{\varepsilon}}{\varepsilon^{2}}\right)+\mathcal{B}\left(r_{\varepsilon, \delta}-\frac{1}{\left|\Omega_{\varepsilon}\right|} \int_{\Omega_{\varepsilon}} r_{\varepsilon, \delta}\right) .
$$

From which we deduce that

$$
\left\|\partial_{t} v^{\delta}\right\|_{L_{T}^{2}\left(\Omega_{\varepsilon}\right)} \leq \frac{C}{\varepsilon^{2}}\left\|\rho_{\varepsilon, \delta} u_{\varepsilon}\right\|_{L_{T}^{2}\left(\Omega_{\varepsilon}\right)}+\frac{C}{\varepsilon}\left\|r_{\varepsilon, \delta}\right\|_{L_{T}^{2}\left(L^{\frac{2 \gamma}{2+\gamma}}\left(\Omega_{\varepsilon}\right)\right)}
$$

and then, we can send $\delta$ to 0 .

Now, we need an other estimate for $\widehat{p}_{\varepsilon}$. Let $F_{\varepsilon} \in \mathcal{D}^{\prime}((0, T) \times \Omega)$ be defined by

$$
\left\langle F_{\varepsilon}, v\right\rangle_{\mathcal{D}^{\prime}, \mathcal{D}(\Omega)}=\left\langle\nabla p_{\varepsilon}, R_{\varepsilon}(v)\right\rangle_{\mathcal{D}^{\prime}, \mathcal{D}\left(\Omega_{\varepsilon}\right)} \quad \forall v \in \mathcal{D}((0, T) \times(\Omega)) .
$$

Hence,

$$
\begin{aligned}
\left\langle F_{\varepsilon}, v\right\rangle_{\mathcal{D}^{\prime}, \mathcal{D}(\Omega)}= & +\varepsilon^{2} \int_{0}^{T} \int_{\Omega_{\varepsilon}} \rho_{\varepsilon} u_{\varepsilon} \partial_{t} R_{\varepsilon}(v)+\int_{0}^{T} \int_{\Omega_{\varepsilon}} \rho_{\varepsilon} u_{\varepsilon} \otimes u_{\varepsilon}: \nabla R_{\varepsilon}(v) \\
& -\int_{0}^{T} \int_{\Omega_{\varepsilon}} \mu \nabla u_{\varepsilon} \cdot \nabla R_{\varepsilon}(v)-\int_{0}^{T} \int_{\Omega_{\varepsilon}} \xi \operatorname{div} u_{\varepsilon} \operatorname{div} R_{\varepsilon}(v) \\
& +\int_{0}^{T} \int_{\Omega_{\varepsilon}}\left(\rho_{\varepsilon} f+g\right) R_{\varepsilon}(v) \\
= & \sum_{i=1}^{5} I_{i}
\end{aligned}
$$

Again, we want to estimate each one of these five terms. We have

$$
\begin{aligned}
\left|I_{1}\right| & \leq C \varepsilon^{2}\left\|\rho_{\varepsilon} u_{\varepsilon}\right\|_{L_{T}^{2}\left(\Omega_{\varepsilon}\right)}\left\|\partial_{t} R_{\varepsilon}(v)\right\|_{L_{T}^{2}\left(\Omega_{\varepsilon}\right)} \leq C \varepsilon^{3}\|v\|_{H_{0}^{1}((0, T) \times \Omega)} \\
\left|I_{2}\right| & \leq C\left\|\rho_{\varepsilon} u_{\varepsilon}\right\|_{L_{T}^{2}\left(\Omega_{\varepsilon}\right)}\left\|u_{\varepsilon}\right\|_{L_{T}^{2}\left(L^{\frac{2 \gamma}{\gamma-2}}\left(\Omega_{\varepsilon}\right)\right)}\left\|\nabla R_{\varepsilon}(v)\right\|_{L_{T}^{\infty}\left(L^{\gamma}(\Omega)\right)} \leq C \varepsilon\|v\|_{L_{T}^{\infty}\left(W_{0}^{1, \gamma}(\Omega)\right)} \\
\left|I_{3}+I_{4}\right| & \leq C\left\|\nabla u_{\varepsilon}\right\|_{L_{T}^{2}\left(\Omega_{\varepsilon}\right)}\left\|\nabla R_{\varepsilon}(v)\right\|_{L_{T}^{2}\left(\Omega_{\varepsilon}\right)} \leq C\left[\|v\|_{L_{T}^{2}(\Omega)}+\varepsilon\|\nabla v\|_{L_{T}^{2}(\Omega)}\right] \\
\left|I_{5}\right| & \leq C\left[\|v\|_{L_{T}^{2}(\Omega)}+\varepsilon\|\nabla v\|_{L_{T}^{2}(\Omega)}\right] .
\end{aligned}
$$

Finally, we deduce that $F_{\varepsilon} \in L^{2}((0, T) \times \Omega)+\varepsilon\left[H^{-1}((0, T) \times \Omega)+L^{1}\left(0, T ; W^{-1, \gamma^{\prime}}(\Omega)\right)\right]$. Extracting a subsequence and passing to the limit, we deduce the existence of some $F \in L^{2}((0, T) \times \Omega)$ such that $F_{\varepsilon}$ converges weakly to $F$. Moreover for all $v \in \mathcal{D}((0, T) \times(\Omega))$ such that $\operatorname{div} v=0$, we have $\left\langle F_{\varepsilon}, v\right\rangle_{\mathcal{D}^{\prime}, \mathcal{D}(\Omega)}=0$ and hence $\langle F, v\rangle_{\mathcal{D}^{\prime}, \mathcal{D}(\Omega)}=0$. Using the result of [15], we know that $F_{\varepsilon}=\nabla \widehat{p}_{\varepsilon}$. Then, we deduce the existence of some $p \in L_{T}^{2}\left(H^{1}(\Omega)\right)$ such that $F=\nabla p$ and $\widehat{p}_{\varepsilon}$ converges weakly to $p$. As in the previous section, we want to prove that $\widehat{\rho}_{\varepsilon}$ converges strongly in $L_{T}^{2}(\Omega)$ to some $\rho$. To this end, we will split $F_{\varepsilon}=\nabla \widehat{p}_{\varepsilon}$ into three parts $F_{\varepsilon}=F_{\varepsilon 1}+F_{\varepsilon 2}+F_{\varepsilon 3}=\nabla \widehat{p}_{\varepsilon 1}+\nabla \widehat{p}_{\varepsilon 2}+\nabla \widehat{p}_{\varepsilon 3}$, where

$$
\begin{aligned}
& p_{\varepsilon 1}=S_{\varepsilon}\left(\rho_{\varepsilon} f+g+\mu \Delta u_{\epsilon}+\xi \nabla \operatorname{div} u_{\epsilon}\right) \\
& p_{\varepsilon 2}=-S_{\varepsilon}\left(\operatorname{div}\left(\rho_{\epsilon} u_{\epsilon} \otimes u_{\varepsilon}\right)\right) \\
& p_{\varepsilon 3}=-S_{\varepsilon}\left(\varepsilon^{2} \partial_{t} \rho_{\epsilon} u_{\varepsilon}\right) .
\end{aligned}
$$


Hence, $p_{\varepsilon 1}+p_{\varepsilon 2}+p_{\varepsilon 3}=\rho_{\varepsilon}^{\gamma}-\frac{1}{\left|\Omega_{\varepsilon}\right|} \int_{\Omega_{\varepsilon}} \rho_{\varepsilon}^{\gamma}$. We also define $w_{\varepsilon 1}, w_{\varepsilon 2}, w_{\varepsilon 3}$ such that $\operatorname{div} w_{\varepsilon 1}=\operatorname{div} w_{\varepsilon 2}=\operatorname{div} w_{\varepsilon 3}=0$, $w_{\varepsilon 1}, w_{\varepsilon 2}$ and $w_{\varepsilon 3}$ vanish on the boundary $\partial \Omega_{\varepsilon}$ and

$$
\begin{aligned}
& -\Delta w_{\varepsilon 1}+\nabla p_{\varepsilon 1}=\rho_{\varepsilon} f+g+\mu \Delta u_{\epsilon}+\xi \nabla \operatorname{div} u_{\epsilon} \\
& -\Delta w_{\varepsilon 2}+\nabla p_{\varepsilon 2}=-\operatorname{div}\left(\rho_{\epsilon} u_{\epsilon} \otimes u_{\varepsilon}\right) \\
& -\Delta w_{\varepsilon 3}+\nabla p_{\varepsilon 3}=-\varepsilon^{2} \partial_{t} \rho_{\epsilon} u_{\varepsilon} .
\end{aligned}
$$

Using the regularity estimates recalled in Lemma 1.7 and an extension procedure as in (73), we deduce that $\widehat{p}_{\varepsilon 1} \in \varepsilon L_{T}^{2}(\Omega)+L_{T}^{2}\left(H^{1}(\Omega)\right), \widehat{p}_{\varepsilon 2} \in \varepsilon^{1-\alpha} L_{T}^{2}\left(L^{\frac{N}{N-1}}(\Omega)\right)$ and $p_{\varepsilon 3} \in \varepsilon^{3} H^{-1}\left(0, T ; H^{1}\left(\Omega_{\varepsilon}\right)\right)$, where $\alpha=\left|\frac{N}{2}-\frac{N}{N}\right|$, namely $\alpha=0$ if $N=2$ and $\alpha=\frac{1}{2}$ if $N=3$. We point out that the advantage of working with $p_{\varepsilon 3}$ instead of $\widehat{p}_{\varepsilon 3}$ is that we lose some regularity for $\widehat{p}_{\varepsilon 3}$ since $\widehat{p}_{\varepsilon 3} \in \varepsilon^{3} H^{-1}\left(0, T ; H^{1}\left(\Omega_{\varepsilon}\right)\right)+\varepsilon^{4} H^{-1}\left(0, T ; L^{2}\left(\Omega_{\varepsilon}\right)\right)$.

Now, let us explain the rest of the proof. From the bounds we have for $\widehat{p}_{\varepsilon 1}, \widehat{p}_{\varepsilon 2}$ and $\widehat{p}_{\varepsilon 3}$, we deduce that $\widehat{p}_{\varepsilon 1}$ converges weakly to $p-\frac{1}{|\Omega|} \int_{\Omega} p$ and that $\widehat{p}_{\varepsilon 2}$ and $\widehat{p}_{\varepsilon 3}$ converges weakly to 0 . Using (as in the last section) that $\partial_{t} \widetilde{\rho}_{\varepsilon}$ is bounded in $L^{1}\left(0, T ; W^{-1,1}(\Omega)\right)$, we can pass to the limit in the product $\widetilde{\rho}_{\varepsilon} \widehat{p}_{\varepsilon 1}$ and deduce that $\widetilde{\rho}_{\varepsilon} \widehat{p}_{\varepsilon 1}$ converges weakly to $\theta \rho p$ (see Lem. 5.1 of [14] ).

Moreover, using that $\widehat{p}_{\varepsilon 2} \in \varepsilon^{1-\alpha} L_{T}^{2}\left(L^{\frac{N}{N-1}}(\Omega)\right)$ and that $\widetilde{\rho}_{\varepsilon}$ is bounded in $L_{T}^{\infty}\left(L^{N}(\Omega)\right)$, we deduce that $\widetilde{\rho}_{\varepsilon} \widehat{p}_{\varepsilon 2}$ converges to 0 . Finally, to prove that $\widetilde{\rho}_{\varepsilon} \widehat{p}_{\varepsilon 3}$ converges weakly to 0 , we will use the bound on $p_{\varepsilon 3}$ which yields the existence of some $g_{\varepsilon} \in \varepsilon^{3} L^{2}\left(0, T ; H^{1}\left(\Omega_{\varepsilon}\right)\right)$ such that $p_{\varepsilon 3}=\partial_{t} g_{\varepsilon}$. Next, for all $\phi \in \mathcal{D}((0, T) \times \Omega)$, we have

$$
\begin{aligned}
\int_{0}^{T} \int_{\Omega} \widetilde{\rho}_{\varepsilon} \widehat{p}_{\varepsilon 3} \phi & =\int_{0}^{T} \int_{\Omega_{\varepsilon}}-\partial_{t} \rho_{\varepsilon} g_{\varepsilon} \phi-\rho_{\varepsilon} g_{\varepsilon} \partial_{t} \phi \\
& =\int_{0}^{T} \int_{\Omega_{\varepsilon}}-\rho_{\varepsilon} \frac{u_{\varepsilon}}{\varepsilon^{2}} \cdot \nabla g_{\varepsilon} \phi-\rho_{\varepsilon} \frac{u_{\varepsilon}}{\varepsilon^{2}} \cdot \nabla \phi g_{\varepsilon}-\rho_{\varepsilon} g_{\varepsilon} \partial_{t} \phi
\end{aligned}
$$

which goes to 0 when $\varepsilon$ goes to 0 . Putting the above results together, we deduce that

$$
\widetilde{\rho}_{\varepsilon}\left(\widehat{p}_{\varepsilon}-\frac{1}{|\Omega|} \int_{\Omega} \widehat{p}_{\varepsilon}\right) \rightarrow \theta \rho\left(p-\frac{1}{|\Omega|} \int_{\Omega} p\right)
$$

weakly in $L^{1}((0, T) \times \Omega)$. Besides, using that $\int_{\Omega} \widehat{p}_{\varepsilon}$ is independent of the space variable and that $\widetilde{\rho}_{\varepsilon}$ has some compactness in time, we know that $\widetilde{\rho}_{\varepsilon} \int_{\Omega} \widehat{p}_{\varepsilon}$ converges weakly to $\theta \rho \int_{\Omega} p$ and hence

$$
\widetilde{\rho}_{\varepsilon} \widehat{p}_{\varepsilon} \rightarrow \theta \rho p
$$

weakly. Now, using the same steps as in the last section, we can deduce that $\widehat{\rho}_{\varepsilon}$ converges strongly to $\rho$ in $L_{T}^{\gamma+1}(\Omega)$ and that $\widehat{p}_{\varepsilon}$ converges strongly to $p=\rho^{\gamma}$ in $L_{T}^{\frac{\gamma+1}{\gamma}}(\Omega)$. We point out that the decomposition of $p_{\varepsilon}$ into $p_{\varepsilon}=p_{\varepsilon 1}+p_{\varepsilon 2}+p_{\varepsilon 3}$ can also be used to deduce the $L^{\gamma+1}$ bound for $\rho_{\varepsilon}$.

The second part of the proof consists in the passing to the limit in $\frac{\widetilde{u}_{\varepsilon}}{\varepsilon^{2}}$. The argument will be a slightly different from the one given in the last section as far as the regularization procedure is concerned. Using that $\widehat{\rho}_{\varepsilon}$ converges strongly in $L_{T}^{\gamma+1}(\Omega)$ to some $\rho$, we introduce some direct regularization of $\rho_{\varepsilon}$ by taking $\rho_{\varepsilon, \eta}=\widehat{\rho}_{\varepsilon}^{*}{ }_{x} \chi_{\eta}$ where we have prolonged $\widehat{\rho}_{\varepsilon}$ by 0 outside the domain $\Omega$, we deduce that

$$
\| \widehat{\rho}_{\varepsilon}-\left.\rho_{\varepsilon, \eta}\right|_{L_{T}^{\gamma+1}(\Omega)}
$$

goes to 0 when $\eta$ goes to 0 uniformly with respect to $\varepsilon$. 
For all $\phi \in \mathcal{D}((0, T) \times \Omega)$, we take $\rho_{\varepsilon, \eta} v_{k}^{\varepsilon} \phi$ as a test function in (63)

$$
\begin{aligned}
\int_{0}^{T} \int_{\Omega_{\varepsilon}} \mu \nabla u_{\varepsilon} \cdot \nabla\left(\rho_{\varepsilon, \eta} v_{k}^{\varepsilon} \phi\right)= & \int_{0}^{T} \int_{\Omega}\left(\rho_{\varepsilon, \eta} v_{k}^{\varepsilon} \phi\right)\left(\rho_{\varepsilon} f+g\right) \\
& +\int_{0}^{T} \int_{\Omega} \operatorname{div}\left(\rho_{\varepsilon, \eta} v_{k}^{\varepsilon} \phi\right)\left(\widehat{\rho_{\varepsilon}^{\gamma}}-\xi \operatorname{div} u_{\varepsilon}\right) . \\
& +\int_{0}^{T} \int_{\Omega} \varepsilon^{2} \widetilde{\rho}_{\varepsilon} \widetilde{u}_{\varepsilon} \partial_{t}\left(\rho_{\varepsilon, \eta} v_{k}^{\varepsilon} \phi\right)+\widetilde{\rho}_{\varepsilon} \widetilde{u}_{\varepsilon} \otimes \widetilde{u}_{\varepsilon}: \nabla\left(\rho_{\varepsilon, \eta} v_{k}^{\varepsilon} \phi\right) .
\end{aligned}
$$

In the right hand side, we have replaced the integrations over $\Omega_{\varepsilon}$ by integration over $\Omega$ since $v_{k}^{\varepsilon}$ and $\operatorname{div}\left(v_{k}^{\varepsilon}\right)$ vanish on $\Omega-\Omega_{\varepsilon}$ and $\rho_{\varepsilon}$ and $u_{\varepsilon}$ have been prolonged by $\widetilde{\rho}_{\varepsilon}$ and $\widetilde{u}_{\varepsilon}$. For all fixed $\eta$, we can pass to the limit in $(83)$ and recover

$$
\begin{aligned}
\lim _{\varepsilon \rightarrow 0} \int_{0}^{T} \int_{\Omega_{\varepsilon}} \mu \nabla u_{\varepsilon} \cdot \nabla\left(\rho_{\varepsilon, \eta} v_{k}^{\varepsilon} \phi\right)= & \int_{0}^{T} \int_{\Omega}\left(\rho_{x}^{*} \chi_{\eta} \phi \bar{A} e_{k}\right)(\rho f+g) \\
& +\int_{0}^{T} \int_{\Omega} \nabla\left(\rho *_{x} \chi_{\eta}\right) \cdot \bar{A} e_{k} \phi p+\left(\rho{ }_{x}^{*} \chi_{\eta}\right) \bar{A} e_{k} \cdot \nabla \phi p .
\end{aligned}
$$

On the other hand, using that $-\varepsilon^{2} \Delta v_{k}^{\varepsilon}+\varepsilon \nabla q_{k}^{\varepsilon}=e_{k}$ in $\Omega_{\varepsilon}$ and taking $\rho_{\varepsilon, \eta} \frac{u_{\varepsilon}}{\varepsilon^{2}} \phi$ as a test function, we get as in the last section that

$$
\int_{0}^{T} \int_{\Omega_{\varepsilon}} \nabla v_{k}^{\varepsilon} \cdot \nabla\left(\rho_{\varepsilon, \eta} u_{\varepsilon} \phi\right)+\frac{1}{\varepsilon} \int_{0}^{T} \int_{\Omega_{\varepsilon}} \rho_{\varepsilon, \eta} u_{\varepsilon} \nabla q_{k}^{\varepsilon} \phi=\int_{0}^{T} \int_{\Omega_{\varepsilon}} \rho_{\varepsilon, \eta} \frac{u_{\varepsilon} e_{k}}{\varepsilon^{2}} \phi .
$$

Arguing as in the last section (the definition of $\rho_{\varepsilon, \eta}$ is not the same by the estimates are the same), we get

$$
\int_{0}^{T} \int_{\Omega}\left(\rho \underset{x}{*} \chi_{\eta}\right) \bar{A} e_{k} \cdot(\rho f+g-\nabla p) \phi=\mu \int_{0}^{T} \int_{\Omega}\left(\rho{ }_{x}^{*} \chi_{\eta}\right) u e_{k} \phi+\alpha(\eta)
$$

where we have performed an integration by parts in (84). Sending $\eta$ to 0 and using that $p=\rho^{\gamma} \in L_{T}^{2}\left(H^{1}\right)$ and that $\rho \in L_{T}^{\gamma+1}(\Omega)$, we deduce that

$$
\int_{0}^{T} \int_{\Omega} \rho \bar{A} e_{k} \cdot\left(\rho f+g-\nabla \rho^{\gamma}\right) \phi=\mu \int_{0}^{T} \int_{\Omega} \rho u e_{k} \phi
$$

Hence, we deduce that

$$
\rho u=\frac{1}{\mu} \bar{A}\left(\rho^{2} f+\rho g-\frac{\gamma}{\gamma+1} \nabla \rho^{\gamma+1}\right) .
$$

The rest of the proof follows the same steps as in the last section.

\section{Acoustics in porous medium}

The last model we want to investigate is the following linearized model

$$
\left\{\begin{array}{l}
\partial_{t} p_{\epsilon}+\operatorname{div} u_{\epsilon}=0 \\
\partial_{t} u_{\epsilon}-\varepsilon^{2} \mu \Delta u_{\epsilon}-\varepsilon^{2} \xi \nabla \operatorname{div} u_{\epsilon}+\nabla p_{\varepsilon}=g_{\varepsilon} \\
u_{\varepsilon}=0 \text { on } \partial \Omega_{\varepsilon} \\
u_{\varepsilon}(t=0)=a_{\varepsilon}(x) \quad p_{\varepsilon}(t=0)=b_{\varepsilon}(x) .
\end{array}\right.
$$


This system describes small perturbations of a compressible fluid at rest. The perturbations are assumed to be small enough to be studied in a linearized framework. The Mach number is assumed to be of order one which means that we can take $p_{\varepsilon}=\rho_{\varepsilon}$.

\subsection{Statement of the result}

We consider a sequence of solutions $\left(u_{\varepsilon}, p_{\varepsilon}\right)$ of $(89)$ such that $a_{\varepsilon} \in L^{2}\left(\Omega_{\varepsilon}\right), b_{\varepsilon} \in L^{2}\left(\Omega_{\varepsilon}\right)$ and $g_{\varepsilon} \in L_{T}^{2}\left(L^{2}\left(\Omega_{\varepsilon}\right)\right)$. Hence, $\left(u_{\varepsilon}, p_{\varepsilon}\right)$ satisfies the following energy estimate

$$
\int_{\Omega_{\varepsilon}} \frac{p_{\varepsilon}^{2}(t)}{2}+\frac{\left|u_{\varepsilon}^{2}(t)\right|}{2}+\int_{0}^{t} \int_{\Omega_{\varepsilon}} \varepsilon^{2} \mu\left|\nabla u_{\varepsilon}\right|^{2}+\varepsilon^{2} \xi\left(\operatorname{div} u_{\varepsilon}\right)^{2}=\int_{\Omega_{\varepsilon}} \frac{b_{\varepsilon}^{2}}{2}+\frac{\left|a_{\varepsilon}^{2}(t)\right|}{2}+\int_{0}^{t} \int_{\Omega_{\varepsilon}} g_{\varepsilon} \cdot u_{\varepsilon} .
$$

The homogenized problem can be written in two different ways. One can either write a two-scale homogenized equation or an integro-differential equation. In the following presentation, we will only discuss the two-scale homogenized equation and we will come back to the relation with the integro-differential equation in a forthcoming work [16]. To write the two-scale homogenized equation we need the notion of two-scale convergence. We will not recall the definition here and refer to Nguetseng [18,19] and to Allaire [2]. In the sequel, we will only study the oscillations in the space variable and so the two-scale convergence refers only to the space variable. In [16], we will also deal with the time oscillations. We have the following result

Theorem 4.1. The extension $\left(\widetilde{u}_{\varepsilon}, \widehat{p}_{\varepsilon}\right)$ two-scale converges to the unique solution $(u(t, x, y), p(t, x))$ of the following two-scale problem

$$
\left\{\begin{array}{l}
\partial_{t} u+\nabla_{y} p_{1}+\nabla_{x} p-\Delta_{y y} u=\mathcal{P} g \quad \text { in } \Omega \times \mathcal{Y}_{f} \\
\theta \partial_{t} p+\operatorname{div}_{x}\left[\int_{\mathcal{Y}} u(x, y) \mathrm{d} y\right]=0 \quad \text { in } \Omega \\
\operatorname{div}_{y} u(x, y)=0 \text { in } \Omega \times \mathcal{Y}_{f} \\
{\left[\int_{\mathcal{Y}} u(x, y) \mathrm{d} y\right] . n=0 \quad \text { on } \quad \partial \Omega} \\
u(t, x, y)=0 \text { in } \Omega \times \mathcal{Y}_{s} \quad \text { and } y \mapsto u, p_{1} \text { are } \mathcal{Y}-\text { periodic } \\
u(t=0)=\mathcal{P} a(x, y) \quad \text { and } p(t=0)=b(x)
\end{array}\right.
$$

where $p_{1}$ is the Lagrange multiplier associated with the constraint $\operatorname{div}_{y} u(x, y)=0, a(x, y)$ is the two-scale limit of $a_{\varepsilon}, b(x)$ is the weak limit of $b_{\varepsilon}$ and $g$ is two-scale limit of $g_{\varepsilon}$. Here, $\mathcal{P}$ (which is an operator acting on the $y$ variable) denotes the projection on divergence-free function of $y$, namely for all $v(y) \in L^{2}\left(\mathcal{Y}_{f}\right)$ and $\mathcal{Y}_{\text {-periodic, }}$ $\mathcal{P} v$ is such that $\mathcal{P} v=v-\nabla q(y), \operatorname{div} \mathcal{P} v=0 \mathcal{P} v \cdot n=0$ on $\partial \mathcal{Y}_{s}$. The system (91) is very similar to the system obtained by Allaire [3] where the unsteady incompressible Stokes equation was studied.

Proof. We will only sketch the proof which follows the same steps as the proof for the unsteady incompressible Stokes equation given in [3].

Lemma 4.2. There exists $\left.u(t, x, y) \in L^{\infty}\left(0, T ; L^{2}(\Omega \times \mathcal{Y})\right) \cap L^{2}(0, T) \times \Omega ; H^{1}(\mathcal{Y})\right)$ and $p(t, x) \in L^{\infty}\left(0, T ; L^{2}(\Omega)\right)$ such that, up to a subsequence, the sequences $\widetilde{u}_{\varepsilon}, \varepsilon \nabla \widetilde{u}_{\varepsilon}$ and $\partial_{t} \widetilde{u}_{\varepsilon}$ two-scale converge to $u, \nabla_{y} u$ and $\partial_{t} u$. Moreover, $u$ is such that for all $t$,

$$
\operatorname{div}_{y} u=0 \quad \text { in } \Omega \times \mathcal{Y}, \quad \text { and } \quad u=0 \quad \text { in } \Omega \times \mathcal{Y}_{s}
$$

Proof of the lemma. By the two-scale convergence, there exist four functions $u, V, w$ and $p$ depending on $t$, $x$ and $y$ such that up to the extraction of a subsequence, $\widetilde{u}_{\varepsilon}, \varepsilon \nabla \widetilde{u}_{\varepsilon}, \partial_{t} \widetilde{u}_{\varepsilon}$ and $\widehat{p}_{\varepsilon}$ two-scale converge to $u, V, w$ 
and $p$, namely

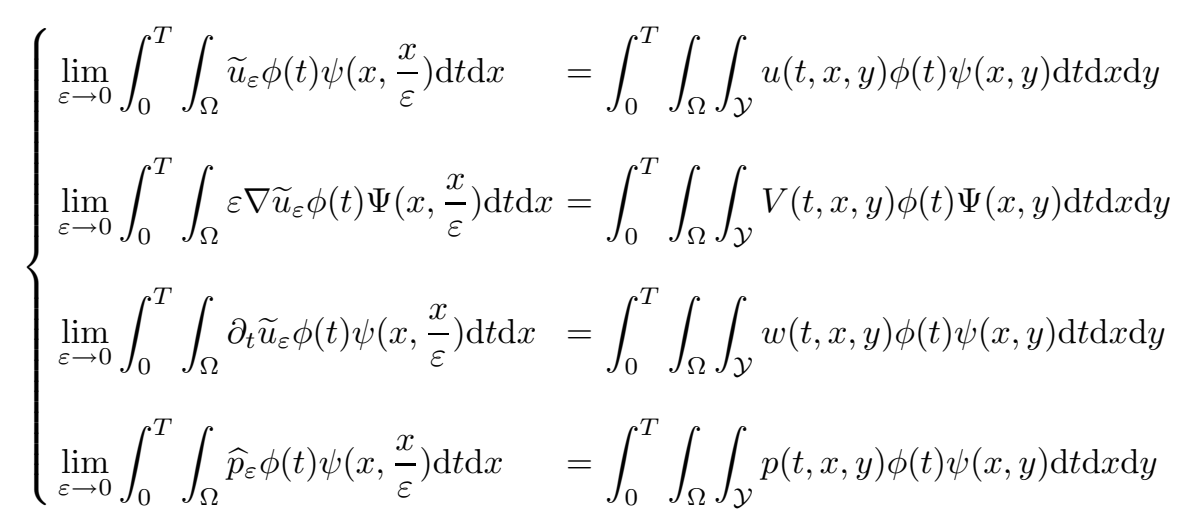

for any $\phi \in \mathcal{D}(0, T), \psi(x, y), \Psi(x, y) \in C(\bar{\Omega} \times \mathcal{Y})$. Integrating by parts and passing to the limit in the second and third equations of (93), we deduce that $\nabla u=V$ and that $\partial_{t} u=w$. To prove that $u=0$ in $\Omega \times \mathcal{Y}_{s}$, we use test functions that vanish on $\Omega \times \mathcal{Y}_{f}$. To prove that $\operatorname{div}_{y} u=0$, we take $\nabla_{y} \psi(x, y)+\varepsilon \nabla_{x} \psi(x, y)$ as a test function and then integrate by parts and use the conservation of mass equation. Finally, to prove that $p$ does not depends on $y$, we use $\varepsilon \phi(t) \psi\left(x, \frac{x}{\varepsilon}\right)$ as a test function in the momentum equation, where $\phi(x, y) \in \mathcal{D}\left(\Omega \times \mathcal{Y}_{f}\right)$ is periodic in $y$.

Now, we want to pass to the limit in the conservation of mass as well as in the momentum equation. For all $\psi(x) \in C^{\infty}(\bar{\Omega})$ and all $\phi \in C_{0}^{\infty}([0, T))$, we have

$$
\int_{0}^{T} \int_{\Omega} \widetilde{p}_{\varepsilon} \psi(x) \partial_{t} \phi(t)+\widetilde{u}_{\varepsilon} \cdot \nabla \psi \phi=-\int_{\Omega} \widetilde{b}_{\varepsilon} \psi(x) .
$$

Passing to the weak limit, we deduce that

$$
\int_{0}^{T} \int_{\Omega} \theta p \psi(x) \partial_{t} \phi(t)+\left[\int_{\mathcal{Y}} u(x, y) \mathrm{d} y\right] \cdot \nabla \psi \phi=-\int_{\Omega} \theta b \psi(x) .
$$

Hence, we get

$$
\left\{\begin{array}{l}
\theta \partial_{t} p+\operatorname{div}_{x}\left[\int_{\mathcal{Y}} u(x, y) \mathrm{d} y\right]=0 \quad \text { in } \quad \Omega \\
{\left[\int_{\mathcal{Y}} u(x, y) \mathrm{d} y\right] \cdot n=0 \quad \text { on } \quad \partial \Omega \text { and } p(t=0)=b(x)}
\end{array}\right.
$$

To pass to the limit in the momentum equation, we take $\phi(t) \psi\left(x, \frac{x}{\varepsilon}\right)$ as a test function, where $\psi(x, y) \in \mathcal{D}\left(\Omega \times \mathcal{Y}_{f}\right)$ is periodic in $y$ and $\operatorname{div}_{y} \psi=0$. Integrating by parts an passing to the limit, we get

$$
\begin{gathered}
-\int_{0}^{T} \int_{\Omega} \int_{\mathcal{Y}} u(t, x, y) \cdot \partial_{t} \phi \psi(x, y) \mathrm{d} t \mathrm{~d} x \mathrm{~d} y-\int_{0}^{T} \int_{\Omega} \int_{\mathcal{Y}} p \phi \operatorname{div}_{x} \psi(x, y)+\int_{0}^{T} \int_{\Omega} \int_{\mathcal{Y}} \nabla_{y} u \cdot \nabla_{y} \psi(x, y) \\
=\int_{0}^{T} \int_{\Omega} \int_{\mathcal{Y}} g(t, x, y) \phi(t) \psi(x, y) \mathrm{d} t \mathrm{~d} x \mathrm{~d} y-\int_{\Omega} \int_{\mathcal{Y}} a(x, y) \phi(0) \psi(x, y) .
\end{gathered}
$$

From which we deduce the existence of $p_{1}$ periodic in $y$ such that the first equation in (91) holds. 


\section{REFERENCES}

[1] G. Allaire, Homogenization of the Stokes flow in a connected porous medium. Asymptot. Anal. 2 (1989) 203-222.

[2] G. Allaire, Homogenization and two-scale convergence. SIAM J. Math. Anal. 23 (1992) 1482-1518.

[3] G. Allaire, Homogenization of the unsteady Stokes equations in porous media, in Progress in partial differential equations: Calculus of variations, applications, Pont-à-Mousson, 1991. Longman Sci. Tech., Harlow (1992) 109-123.

[4] A. Bensoussan, J.-L. Lions and G. Papanicolaou, Asymptotic analysis for periodic structures. North-Holland Publishing Co., Amsterdam (1978).

[5] M.E. Bogovskilu, Solutions of some problems of vector analysis, associated with the operators div and grad, in Theory of cubature formulas and the application of functional analysis to problems of mathematical physics. Akad. Nauk SSSR Sibirsk. Otdel. Inst. Mat., Novosibirsk (1980) 5-40, 149.

[6] L. Cattabriga, Su un problema al contorno relativo al sistema di equazioni di Stokes. Rend. Sem. Mat. Univ. Padova 31 (1961) 308-340.

[7] H. Darcy, Les fontaines publiques de la ville de Dijon. Dalmont Paris (1856).

[8] J.I. Díaz, Two problems in homogenization of porous media, in Proc. of the Second International Seminar on Geometry, Continua and Microstructure, Getafe, 1998, Vol. 14 (1999) 141-155.

[9] E. Feireisl, On compactness of solutions to the compressible isentropic Navier-Stokes equations when the density is not square integrable. Comment. Math. Univ. Carolin. 42 (2001) 83-98.

[10] G.P. Galdi, An introduction to the mathematical theory of the Navier-Stokes equations, Vol. I. Springer-Verlag, New York (1994). Linearized steady problems.

[11] J.-L. Lions, Quelques méthodes de résolution des problèmes aux limites non linéaires. Dunod (1969).

[12] J.-L. Lions, Some methods in the mathematical analysis of systems and their control. Kexue Chubanshe (Science Press), Beijing (1981).

[13] P.-L. Lions, Mathematical topics in fluid mechanics, Vol. 1. The Clarendon Press Oxford University Press, New York (1996). Incompressible models, Oxford Science Publications.

[14] P.-L. Lions, Mathematical topics in fluid mechanics, Vol. 2. The Clarendon Press Oxford University Press, New York (1998). Compressible models, Oxford Science Publications.

[15] R. Lipton and M. Avellaneda, Darcy's law for slow viscous flow past a stationary array of bubbles. Proc. Roy. Soc. Edinburgh Sect. A 114 (1990) 71-79.

[16] N. Masmoudi (in preparation).

[17] A. Mikelić, Homogenization of nonstationary Navier-Stokes equations in a domain with a grained boundary. Ann. Mat. Pura Appl. (4) 158 (1991) $167-179$.

[18] G. Nguetseng, A general convergence result for a functional related to the theory of homogenization. SIAM J. Math. Anal. 20 (1989) 608-623.

[19] G. Nguetseng, Asymptotic analysis for a stiff variational problem arising in mechanics. SIAM J. Math. Anal. 21 (1990) 1394-1414.

[20] E. Sánchez-Palencia, Nonhomogeneous media and vibration theory. Springer-Verlag, Berlin (1980).

[21] L. Tartar, Incompressible fluid flow in a porous medium: convergence of the homogenization process, in Nonhomogeneous media and vibration theory, edited by E. Sánchez-Palencia (1980) 368-377.

[22] R. Temam, Navier-Stokes equations and nonlinear functional analysis. Society for Industrial and Applied Mathematics (SIAM), Philadelphia, PA, Second Edition (1995). 